
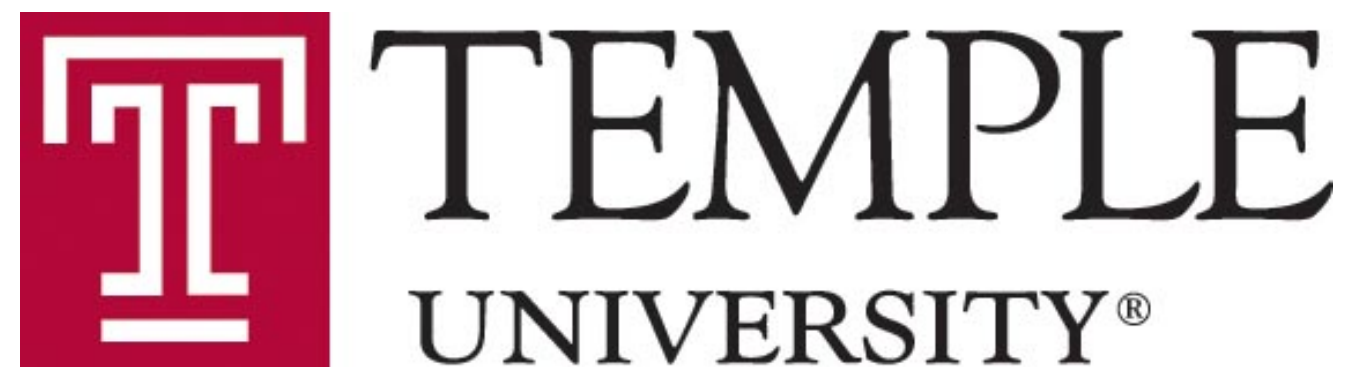

\title{
Difference-in-Differences with Multiple Time Periods and an Application on the Minimum Wage and Employment
}

\author{
Brantly Callaway \\ Department of Economics \\ Temple University \\ Pedro H. C. Sant'Anna \\ Department of Economics \\ Vanderbilt University
}

Department of Economics

DETU Working Paper 18-04

March 2018

1301 Cecil B. Moore Avenue, Philadelphia, PA 19122

http://www.cla.temple.edu/economics/faculty/detu-working-paper-series/ 


\title{
Difference-in-Differences with Multiple Time Periods and
}

\section{an Application on the Minimum Wage and Employment*}

\author{
Brantly Callaway ${ }^{\dagger} \quad$ Pedro H. C. Sant’Anna $a^{\ddagger}$
}

March 23, 2018

\begin{abstract}
Difference-in-Differences (DID) is one of the most important and popular designs for evaluating causal effects of policy changes. In its standard format, there are two time periods and two groups: in the first period no one is treated, and in the second period a "treatment group" becomes treated, whereas a "control group" remains untreated. However, many empirical applications of the DID design have more than two periods and variation in treatment timing. In this article, we consider identification and estimation of treatment effect parameters using DID with (i) multiple time periods, (ii) variation in treatment timing, and (iii) when the "parallel trends assumption" holds potentially only after conditioning on observed covariates. We propose a simple two-step estimation strategy, establish the asymptotic properties of the proposed estimators, and prove the validity of a computationally convenient bootstrap procedure. Furthermore we propose a semiparametric data-driven testing procedure to assess the credibility of the DID design in our context. Finally, we analyze the effect of the minimum wage on teen employment from 2001-2007. By using our proposed methods we confront the challenges related to variation in the timing of the state-level minimum wage policy changes. Open-source software is available for implementing the proposed methods.
\end{abstract}

JEL: C14, C21, C23, J23, J38.

Keywords: Difference-in-Differences, Multiple Periods, Variation in Treatment Timing, PreTesting, Minimum Wage.

*We thank Andrew Goodman-Bacon, Federico Gutierrez, Na'Ama Shenhav, and seminar participants at the 2017 Southern Economics Association for valuable comments. Code to implement the methods proposed in the paper are available in the $\mathrm{R}$ package did which is available on CRAN.

${ }^{\dagger}$ Department of Economics, Temple University. Email: brantly.callaway@temple.edu

${ }^{\ddagger}$ Department of Economics, Vanderbilt University. Email: pedro.h.santanna@vanderbilt.edu 


\section{Introduction}

Difference-in-Differences (DID) has become one of the most popular designs used to evaluate the causal effect of policy interventions. In its canonical format, there are two time periods and two groups: in the first period, no one is treated and in the second period some individuals are treated (the treated group) and some individuals are not (the control group). If, in the absence of treatment, the average outcomes for treated and control groups would have followed parallel paths over time (which is the so-called parallel trends assumption), one can measure the average treatment effect for the treated subpopulation (ATT) by comparing the average change in outcomes experienced by the treated group to the average change in outcomes experienced by the control group. Most methodological extensions of DID methods have been confined to this standard two periods, two groups setup, see e.g. Heckman et al. (1997, 1998), Abadie (2005), Athey and Imbens (2006), Qin and Zhang (2008), Bonhomme and Sauder (2011), de Chaisemartin and D'Haultfœuille (2017), Botosaru and Gutierrez (2017), and Callaway et al. (2018).

Many DID empirical applications, however, deviate from the standard DID setup. For example, half of the articles published in 2014/2015 in the American Economic Review, Quarterly Journal of Economics, and the Journal of Political Economy that used DID methods had more than two time periods and exploited variation in treatment timing. ${ }^{1}$ In these cases, researchers usually consider the following regression model

$$
Y_{i t}=\alpha_{t}+c_{i}+\beta D_{i t}+\theta X_{i}+\epsilon_{i t}
$$

where $Y_{i t}$ is the outcome of interest, $\alpha_{t}$ is a time fixed effect, $c_{i}$ is an individual/group fixed effect, $D_{i t}$ is a treatment indicator that takes value one if an individual $i$ is treated at time $t$ and zero otherwise, $X_{i}$ is a vector of observed characteristics, and $\epsilon_{i t}$ is an error term, and interpret $\beta$ as the causal effect of interest. Despite the popularity of this approach, Wooldridge (2005), Chernozhukov et al. (2013), de Chaisemartin and D'Haultfoeuille (2016), Borusyak and Jaravel (2017), Goodman-Bacon (2017) and Słoczyński (2017) have shown that once one allows for heterogeneous treatment effects, $\beta$ does not represent an easy to interpret average treatment

\footnotetext{
${ }^{1}$ We thank Andrew Goodman-Bacon for sharing with us this statistic.
} 
effect parameter. As a consequence, inference about the effectiveness of a given policy can be misleading when based on such a two-way fixed effects regression model.

In this article we aim to fill this important gap and consider identification and inference procedures for average treatment effects in DID models with $(i)$ multiple time periods, $(i i)$ variation in treatment timing, and (iii) when the parallel trends assumption holds potentially only after conditioning on observed covariates. First, we provide conditions under which the average treatment effect for group $g$ at time $t$ is nonparametrically identified, where a "group" is defined by when units are first treated. We call these causal parameters group-time average treatment effects.

Second, although these disaggregated group-time average treatment effects can be of interest by themselves, in some applications there are perhaps too many of them, potentially making the analysis of the effectiveness of the policy intervention harder, particularly when the sample size is moderate. In such cases, researchers may be interested in summarizing these disaggregated causal effects into a single, easy to interpret, causal parameter. We suggest different ideas for combining the group-time average treatment effects, depending on whether one allows for $(a)$ selective treatment timing, i.e., allowing, for example, the possibility that individuals with the largest benefits from participating in a treatment choose to become treated earlier than those with a smaller benefit; $(b)$ dynamic treatment effects - where the effect of a treatment can depend on the length of exposure to the treatment; or $(c)$ calendar time effects - where the effect of treatment may depend on the time period. Overall, we note that the best way to aggregate the group-time average treatment effects is likely to be application specific. Aggregating group-time parameters is also likely to increase statistical power.

Third, we develop the asymptotic properties for a semiparametric two-step estimator for the group-time average treatment effects, and for the different aggregated causal parameters. Estimating these treatment effects involves estimating a generalized propensity score for each group $g$, and using these to construct appropriate weights for a "long difference" of outcomes. We establish $\sqrt{n}$ consistency and asymptotic normality of our estimators. We propose computationally convenient bootstrapped simultaneous confidence bands that can be used for visualizing estimation uncertainty for the group-time average treatment effects. Unlike traditional pointwise confidence bands, 
our simultaneous confidence bands asymptotically cover the entire path of the group-time average treatment effects with probability $1-\alpha$. Importantly, our inference procedures can accommodate clustering in a relatively straightforward manner.

Finally, it is important to emphasize that all the aforementioned results rely on the fundamentally untestable conditional parallel trends assumption. Nonetheless, we note that if one imposes a slightly stronger conditional parallel trends assumption - namely, that conditional parallel trends holds in all periods, specifically including pre-treatment periods - there are testable implications, provided the availability of more than two time periods. A fourth contribution of this article is to take advantage of this observation and propose a falsification test based on it. Our pre-test for the plausibility of the conditional parallel trends assumption is based on the integrated moments approach, see e.g. Bierens (1982) and Stute (1997), completely avoids selecting tuning parameters, and is fully data-driven. We use results from the empirical processes literature to study the asymptotic properties of our falsification test. In particular, we derive its asymptotic null distribution, prove that it is consistent against fixed nonparametric alternatives, and show that critical values can be computed with the assistance of an easy to implement multiplier-type bootstrap.

We illustrate the appeal of our method by revisiting findings about the effect of the minimum wage on teen employment. Although standard economic theory says that a wage floor should result in lower employment, there is a bulk of literature that finds no disemployment effects of the minimum wage, even when focusing on groups that are most likely to be affected by minimum wage increases such as teenagers or fast-food restaurant employees. Notable among these is the landmark DID paper, Card and Krueger (1994), Dube et al. (2010), among many others; see also Doucouliagos and Stanley (2009), Schmitt (2013), and Belman and Wolfso (2014) for summaries of the literature. There is notable disagreement with this view though. For instance, in a series of work, David Neumark and William Wascher (see e.g. Neumark and Wascher (1992, 2000, 2008), Neumark et al. (2014)), as well as Jardim et al. (2017) provide evidence for the view that increasing the minimum wage decreases employment.

We use data from 2001-2007, where the federal minimum wage was flat at $\$ 5.15$ per hour. Using a period where the federal minimum wage is flat allows for a clear source of identification 
- state level changes in minimum wage policy. However, we also need to confront the issue that states changed their minimum wage policy at different points in time over this period - an issue not encountered in the case study approach to studying the employment effects of the minimum wage. In addition, for states that changed their minimum wage policy in later periods, we can pre-test the parallel trends assumption which serves as a check of the internal consistency of the models used to identify minimum wage effects.

We consider both an unconditional and conditional DID approach to estimating the effect of increasing the minimum wage on teen employment rates. For the unconditional case, we find that increases in the minimum wage tend to decrease teen employment; the effects range from $2.3 \%$ lower teen employment to $13.6 \%$ lower teen employment across groups and time. This result is not surprising; most of the work on the minimum wage that reaches a similar conclusion uses a similar setup. Dube et al. (2010) points out that such negative effects may be spurious given potential violations of the common trend assumption. Indeed, when we tests for the reliability of the unconditional common trend assumption, we reject it. Given that we reject the unconditional DID design, we then follow Dube et al. (2010) proposal and consider a two-way fixed effects regression model with region-year fixed effects. As in Dube et al. (2010), such an estimation strategy finds no adverse effect on teen employment. Nonetheless, one must bear in mind that, as discussed before, such two-way fixed effects regressions may not identify easy to interpret causal parameters. To circumvent this issue, we use our conditional DID approach and find that increasing the minimum wage does tend to decrease teen employment with effects ranging from $0.8 \%$ higher employment (not statistically significant) to $7.3 \%$ lower employment across groups and time. In addition, like Meer and West (2016), we find that the effect of minimum wage increases is dynamic - the effect is increasing in the length of exposure to the minimum wage increase. However, we find evidence against the conditional parallel trends assumption using our pre-test. Thus, our findings should be interpreted with care.

The methodological results in this article are related to other papers in the DID literature. Heckman et al. (1997, 1998), Blundell et al. (2004), Abadie (2005), and, Qin and Zhang (2008) consider identifying assumptions similar to ours, but focus on the standard DID setup of two 
periods, two groups. Our proposal particularly builds on Abadie (2005) as we also adjusts for covariates using propensity score weighting. On top of accounting for variation in treatment timing, another important difference between our proposal and Abadie (2005) is that our estimator is based on stabilized (normalized) weights, whereas his proposed estimator is of the Horvitz and Thompson (1952) type. As the simulations results in Busso et al. (2014) show, stabilized weights can lead to important finite sample improvements when compared to Horvitz and Thompson (1952) type estimators.

Our pre-test for the plausibility of the conditional parallel trends assumption is also related to many papers in the goodness-of-fit literature, including Bierens (1982), Bierens and Ploberger (1997), Stute (1997), Stinchcombe and White (1998), Escanciano (2006a,b, 2008), Sant'Anna (2017), and Sant'Anna and Song (2018); for a recent overview, see González-Manteiga and Crujeiras (2013). Despite the similarities, we seem to be the first to realize that such a procedure could be used to pre-test for the reliability of the conditional parallel trends identification assumption.

The remainder of this article is organized as follows. Section 2 presents our main identification results. We discuss estimation and inference procedures for the treatment effects of interest in Section 3. Section 4 describes our pre-tests for the credibility of the conditional parallel trends assumption. We revisit the effect of minimum wage on employment in Section 5. Section 6 concludes. All proofs are gathered in the Appendix.

\section{Identification}

\section{$2.1 \quad$ Framework}

We first introduce the notation we use throughout the article. We consider the case with $\mathcal{T}$ periods and denote a particular time period by $t$ where $t=1, \ldots, \mathcal{T}$. In a standard DID setup, $\mathcal{T}=2$ and no one is treated in period 1 . Let $D_{t}$ be a binary variable equal to one if an individual is treated in period $t$ and equal to zero otherwise. Also, define $G_{g}$ to be a binary variable that is equal to one if an individual is first treated in period $g$, and define $C$ as a binary variable that is equal to one for individuals in the control group - these are individuals who are never treated so the notation 
is not indexed by time. For each individual, exactly one of the $G_{g}$ or $C$ is equal to one. Denote the generalized propensity score as $p_{g}(X)=P\left(G_{g}=1 \mid X, G_{g}+C=1\right)$. Note that $p_{g}(X)$ indicates the probability that an individual is treated conditional on having covariates $X$ and conditional on being a member of group $g$ or the control group. Finally, let $Y_{t}(1)$ and $Y_{t}(0)$ be the potential outcome at time $t$ with and without treatment, respectively. The observed outcome in each period can be expressed as $Y_{t}=D_{t} Y_{t}(1)+\left(1-D_{t}\right) Y_{t}(0)$.

Given that $Y_{t}(1)$ and $Y_{t}(0)$ cannot be observed for the same individual at the same time, researchers often focus on estimating some function of the potential outcomes. For instance, in the standard DID setup, the most popular treatment effect parameter is the average treatment effect on the treated, denoted by ${ }^{2}$

$$
A T T=\mathbb{E}\left[Y_{2}(1)-Y_{2}(0) \mid G_{2}=1\right]
$$

Unlike the two period and two group case, when there are more than two periods and variation in treatment timing, it is not obvious which is the main causal parameter of interest. Instead, we consider the the average treatment effect for individuals first treated in period $g$ at time period $t$, denoted by

$$
A T T(g, t)=\mathbb{E}\left[Y_{t}(1)-Y_{t}(0) \mid G_{g}=1\right]
$$

We call this causal parameter the group-time average treatment effect. In particular, note that in the classical DID setup, $A T T(2,2)$ collapses to $A T T$.

In this article, we are interested in identifying and estimating $A T T(g, t)$ and functions of $A T T(g, t)$. Towards this end, we impose the following assumptions.

Assumption 1 (Sampling). $\left\{Y_{i 1}, Y_{i 2}, \ldots Y_{i \mathcal{T}}, X_{i}, D_{i 1}, D_{i 2}, \ldots, D_{i \mathcal{T}}\right\}_{i=1}^{n}$ is independent and identically distributed (iid).

Assumption 2 (Conditional Parallel Trends). For all $t=2, \ldots, \mathcal{T}, g=2, \ldots, \mathcal{T}$ such that $g \leq t$,

$$
\mathbb{E}\left[Y_{t}(0)-Y_{t-1}(0) \mid X, G_{g}=1\right]=\mathbb{E}\left[Y_{t}(0)-Y_{t-1}(0) \mid X, C=1\right] \text { a.s.. }
$$

\footnotetext{
${ }^{2}$ Existence of expectations is assumed throughout.
} 
Assumption 3 (Irreversibility of Treatment). For $t=2, \ldots, \mathcal{T}$,

$$
D_{t}=1 \text { implies that } D_{t+1}=1
$$

Assumption 4 (Overlap). For all $g=2, \ldots, \mathcal{T}, P\left(G_{g}=1\right)>0$ and $p_{g}(X)<1$ a.s..

Assumption 1 implies that we are considering the case with panel data. The extension to the case with repeated cross sections is relatively simple and is developed in Appendix B in the Supplementary Appendix.

Assumption 2, which we refer to as the (conditional) parallel trends assumption throughout the paper, is the crucial identifying restriction for our DID model, and it generalizes the two-period DID assumption to the case where it holds in all periods and for all groups; see e.g. Heckman et al. (1997, 1998), Blundell et al. (2004), and Abadie (2005). It states that, conditional on covariates, the average outcomes for the group first treated in period $g$ and for the control group would have followed parallel paths in the absence of treatment. We require this assumption to hold for all groups $g$ and all time periods $t$ such that $g \leq t$; that is, it holds in all periods after group $g$ is first treated. It is important to emphasize that the parallel trends assumption holds only after conditioning on some covariates $X$, therefore allowing for $X$-specific time trends. All of our analysis continues to go through in the case where an unconditional parallel trends assumption holds by simply setting $X=1$.

Assumption 3 states that once an individual becomes treated, that individual will also be treated in the next period. With regards to the minimum wage application, Assumption 3 says that once a state increases its minimum wage above the federal level, it does not decrease it back to the federal level during the analyzed period. Moreover, this assumption is consistent with most DID setups that exploit the enacting of a policy in some location while the policy is not enacted in another location. ${ }^{3}$

Finally, Assumption 4 states that a positive fraction of the population started to be treated in period $g$, and that, for any possible value of the covariates $X$, there is some positive probability

\footnotetext{
${ }^{3}$ One could potentially relax this assumption by forming groups on the basis of having the entire path of treatment status being the same and then perform the same analysis that we do.
} 
that an individual is not treated. ${ }^{4}$ This is a standard covariate overlap condition, see e.g. Heckman et al. (1997, 1998), Blundell et al. (2004), Abadie (2005).

Remark 1. In some applications, eventually all units are treated, implying that $C$ is never equal to one. In such cases one can consider the "not yet treated" $\left(D_{t}=0\right)$ as a control group instead of the "never treated" $(C=1)$. We consider this case in Appendix $C$ in the Supplementary Appendix, which resembles the event study research design, see e.g. Borusyak and Jaravel (2017).

\subsection{Group-Time Average Treatment Effects}

In this section, we introduce the nonparametric identification strategy for the group-time average treatment effect $A T T(g, t)$. Importantly, we allow for arbitrary treatment effect heterogeneity.

Theorem 1. Under Assumptions 1 - 4 and for $2 \leq g \leq t \leq \mathcal{T}$, the group-time average treatment effect for group $g$ in period $t$ is nonparametrically identified, and given by

$$
\operatorname{ATT}(g, t)=\mathbb{E}\left[\left(\frac{G_{g}}{\mathbb{E}\left[G_{g}\right]}-\frac{\frac{p_{g}(X) C}{1-p_{g}(X)}}{\mathbb{E}\left[\frac{p_{g}(X) C}{1-p_{g}(X)}\right]}\right)\left(Y_{t}-Y_{g-1}\right)\right] .
$$

Theorem 1 says that, under Assumptions 1 - 4, a simple weighted average of "long differences" of the outcome variable recovers the group-time average treatment effect. The weights depends on the generalized propensity score $p_{g}(X)$, and are normalized to one. The intuition for the weights is simple. One takes observations from the control group and group $g$, omitting other groups and then weights up observations from the control group that have characteristics similar to those frequently found in group $g$ and weights down observations from the control group that have characteristics that are rarely found in group $g$. Such a reweighting procedures guarantees that the covariates of group $g$ and the control group are balanced. Interestingly, in the standard DID setup of two periods only, $\mathbb{E}\left[p_{2}(X) C /\left(1-p_{2}(X)\right)\right]=\mathbb{E}\left[G_{2}\right]$, and the results of Theorem 1 reduces to Lemma 3.1 in Abadie (2005).

\footnotetext{
${ }^{4}$ In our application on the minimum wage, we must take somewhat more care here as there are some periods where there are no states that increase their minimum wage. In this case, let $\mathcal{G}$ denote the set of first treatment times with $\mathcal{G} \subseteq\{1, \ldots, \mathcal{T}\}$. Then, one can compute $A T T(g, t)$ for groups $g \in \mathcal{G}$ with $g \leq t$. This is a simple complication to deal with in practice, so we consider the notationally more convenient case where there are some individuals treated in all periods (possibly excluding period 1) in the main text of the paper.
} 
To shed light on the role of the "long difference", we give a sketch of how this argument works in the unconditional case, i.e., when $X=1$. Recall that the key identification challenge is for $\mathbb{E}\left[Y_{t}(0) \mid G_{g}=1\right]$ which is not observed when $g \leq t$. Under the parallel trends assumption,

$$
\begin{aligned}
\mathbb{E}\left[Y_{t}(0) \mid G_{g}=1\right] & =\mathbb{E}\left[Y_{t}(0)-Y_{t-1}(0) \mid G_{g}=1\right]+\mathbb{E}\left[Y_{t-1}(0) \mid G_{g}=1\right] \\
& =\mathbb{E}\left[Y_{t}-Y_{t-1} \mid C=1\right]+\mathbb{E}\left[Y_{t-1}(0) \mid G_{g}=1\right]
\end{aligned}
$$

The first term is identified, it is the change in outcomes between $t-1$ and $t$ experienced by the control group. If $g>t-1$, then the last term is identified. If not,

$$
\mathbb{E}\left[Y_{t-1}(0) \mid G_{g}=1\right]=\mathbb{E}\left[Y_{t-1}-Y_{t-2} \mid C=1\right]+\mathbb{E}\left[Y_{t-2}(0) \mid G_{g}=1\right]
$$

which holds under the parallel trends assumption. If $g>t-2$, then every term above is identified. If not, one can proceed recursively in this same fashion until

$$
\mathbb{E}\left[Y_{t}(0) \mid G_{g}=1\right]=\mathbb{E}\left[Y_{t}-Y_{g-1} \mid C=1\right]+\mathbb{E}\left[Y_{g-1} \mid G_{g}=1\right]
$$

implying the result for $\operatorname{ATT}(g, t)$.

One final thing to consider in this section is the case when the parallel trends assumption holds without needing to condition on covariates. In this case, (2.1) simplifies to

$$
A T T(g, t)=\mathbb{E}\left[Y_{t}-Y_{g-1} \mid G_{g}=1\right]-\mathbb{E}\left[Y_{t}-Y_{g-1} \mid C=1\right]
$$

which is simpler than the weighted representation in (2.1) but also implies that all of our results will also cover the unconditional case which is very commonly used in empirical work. We discuss an alternative regression based approach to obtaining $A T T(g, t)$ in Appendix D in the Supplementary Appendix. ${ }^{5}$

\footnotetext{
${ }^{5}$ Unlike the two period, two group case, there does not appear to be any advantage to trying to obtain $A T T(g, t)$ from a regression as it appears to require post-processing the regression output.
} 


\subsection{Summarizing Group-time Average Treatment Effects}

The previous section shows that the group-time average treatment effect $\operatorname{ATT}(g, t)$ is identified for $g \leq t$. These are very useful parameters - they allow one to consider how the effect of treatment varies by group and time. However, in some applications there may be many of them, perhaps too many to easily interpret the effect of a given policy intervention. This section considers ways to aggregate group-time average treatment effects into a few number of interpretable causal effect parameters. In applications, aggregating the group-time average treatment effects is also likely to increase statistical power, reducing estimation uncertainty.

The two simplest ways of combining $A T T(g, t)$ across $g$ and $t$ are

$$
\frac{2}{\mathcal{T}(\mathcal{T}-1)} \sum_{g=2}^{\mathcal{T}} \sum_{t=2}^{\mathcal{T}} \mathbf{1}\{g \leq t\} A T T(g, t) \quad \text { and } \quad \frac{1}{\kappa} \sum_{g=2}^{\mathcal{T}} \sum_{t=2}^{\mathcal{T}} \mathbf{1}\{g \leq t\} A T T(g, t) P(G=g)
$$

where $\kappa=\sum_{g=2}^{\mathcal{T}} \sum_{t=2}^{\mathcal{T}} 1\{g \leq t\} P(G=g)$ (which ensures that the weights on $A T T(g, t)$ in the second term sum to 1). ${ }^{6}$ The first term in (2.3) is just the simple average of $A T T(g, t)$; the second is a weighted average of each $A T T(g, t)$ putting more weight on $A T T(g, t)$ with larger group sizes. As we argue below, neither of the terms in (2.3) are likely to be "appropriate" summary treatment effect measures, except in the particular case where the effect of treatment is homogeneous across groups and time.

In contrast to our approach in this section, the most common approach to estimating the effect of a binary treatment in a panel data setup is to interpret $\beta$ in the following regression as the average treatment effect

$$
Y_{i t}=\alpha_{t}+c_{i}+\beta D_{i t}+\theta X_{i}+\epsilon_{i t}
$$

where $\alpha_{t}$ is a time fixed effect and $c_{i}$ is an individual/group fixed effect. Interestingly, Wooldridge (2005), Chernozhukov et al. (2013), de Chaisemartin and D'Haultfoeuille (2016), Borusyak and Jaravel (2017), Goodman-Bacon (2017) and Słoczyński (2017) have shown that, in general, $\beta$ does not represent an easy to interpret average treatment effect parameter. The results in this section

\footnotetext{
${ }^{6}$ Here we use the shorthand notation $P(G=g)$ to denote $P\left(G_{g}=1 \mid G_{1}+C=0\right)$. Thus, $P(G=g)$ is the probability that an individual is first treated in period $g$ conditional on not being in the control group or in the group first treated in period 1 . Throughout this section, conditional probabilities such as $P(G=g \mid g \leq t)$ also implicitly condition on not being in the control group or in the group first treated in period 1.
} 
can be used in exactly the same setup to identify a single interpretable average treatment effect parameter and, thus, provide a way to circumvent the issues with the more common approach.

In the following, we consider several common cases that are likely to occur in practice: $(a)$ selective treatment timing, (b) dynamic treatment effects, and (c) calendar time effects. We provide some recommendations on constructing interpretable treatment effect parameters under each of these setups. It is worth mentioning that in each of these cases, $\operatorname{ATT}(g, t)$ still provides the average causal effect of the treatment for group $g$ in period $t$; the issue in this section is how to aggregate $A T T(g, t)$ into a smaller number of causal effect parameters.

Selective Treatment Timing In many cases, when to become treated is a choice variable. The parallel trends assumptions does place some restrictions on how individuals select when to be treated. In particular, in order for the path of untreated potential outcomes to be the same for a particular group and the control group, the parallel trends assumption does not permit individuals to select into treatment in period $t$ because they anticipate $Y_{t}(0)$ being small (assuming larger $Y$ is "good"). On the other hand, the parallel trends assumption does not place restrictions on how treated potential outcomes are generated. Thus, our imposed DID assumptions fully allow for individuals to select into treatment on the basis of expected future values of treated potential outcomes.

While some forms of selective treatment timing are permitted under the parallel trends assumption and do not affect identification of group-time average treatment effects, they do have implications for the "best ways" to combine $A T T(g, t)$ into a single, easy to interpret treatment effect parameter. In particular, when there is selective treatment timing, the period when an individual is first treated may provide information about the size of the treatment effect. In such cases, we propose to summarize the causal effect of a policy by first aggregating $A T T(g, t)$ by group, and then combine group average treatment effects based on the size of each group.

More precisely, we first consider

$$
\tilde{\theta}_{S}(g)=\frac{1}{\mathcal{T}-g+1} \sum_{t=2}^{\mathcal{T}} \mathbf{1}\{g \leq t\} \operatorname{ATT}(g, t)
$$


Note that $\tilde{\theta}_{S}(g)$ is the time-averaged treatment effect for individuals in group $g$, i.e., just a timeaverage of each available $A T T(g, t)$ for group $g$. Next, in order to further reduce the dimensionality of $\tilde{\theta}_{S}(g)$, one can average $\tilde{\theta}_{S}(g)$ across groups to get

$$
\theta_{S}=\sum_{g=2}^{\mathcal{T}} \tilde{\theta}_{S}(g) P(G=g)
$$

Note that $\theta_{S}$ appears to be quite similar to the second term in (2.3). The difference is in the weights. The second term in (2.3) puts more weight on groups that are exposed to treatment longer. The weights in (2.4) only depend on group size, not on the number of post-treatment periods available per group. For example, suppose there is positive selective treatment timing so that individuals who are treated earlier experience larger benefits from being treated than those who are treated later. In the presence of selective treatment timing, the approach in (2.3) would tend to overstate the effect of the treatment due to putting more weight on the groups that are treated the longest, which are precisely the ones that experience the largest benefits of being treated. Thus, we argue that, in the presence of selective treatment timing, $\theta_{S}$ in (2.4) is a more natural causal parameter than the second term in (2.3).

Dynamic Treatment Effects In other cases, the effect of a policy intervention may depend on the length of exposure to it. To give some examples, Jacobson et al. (1993) argues that workers that are displaced from their jobs tend to have immediate large earnings effects that get smaller over time, and both the immediate effect and the dynamic effect are of interest. In the case of the minimum wage, Meer and West (2016) argue that increasing the minimum wage leads to lower job creation and thus that the effect of the minimum wage on employment is dynamic - one should expect larger effects in subsequent periods than in the initial period.

In the presence of dynamic treatment effects (but not selective treatment timing), we propose to summarize the effects of the policy by first aggregating $A T T(g, t)$ by the length of exposure to treatment (we denote this by $e$ ), and then (possibly) combining average effects based on length of 
exposure by averaging over different lengths of exposure. That is, we first consider the parameter

$$
\tilde{\theta}_{D}(e)=\sum_{g=2}^{\mathcal{T}} \sum_{t=2}^{\mathcal{T}} \mathbf{1}\{t-g+1=e\} \operatorname{ATT}(g, t) P(G=g \mid t-g+1=e)
$$

which provides the average effect of treatment for individuals that have been treated for exactly $e$ periods. For example, when $e=1$, it averages (based on group size) $A T T(g, t)$ for $g=t$ (groups that have been exposed to treatment for exactly one period). Averaging over all possible values of $e$ results in the parameter

$$
\theta_{D}=\frac{1}{\mathcal{T}-1} \sum_{e=1}^{\mathcal{T}-1} \tilde{\theta}_{D}(e)
$$

The primary difference between $\theta_{D}, \theta_{S}$, and the second term in (2.3) is the weights. Relative to the other parameters, $\theta_{D}$ puts the most weight on $A T T(g, t)$ when $g$ is much less than $t$, which corresponds to large values of $e$, because there are few groups available for large values of $e$. In the absence of selective treatment timing, these groups are informative about the dynamic effects of treatment for all groups. Hence, we argue that $\theta_{D}$ is appealing when treatment effects evolves over time.

Calendar Time Effects In other cases, calendar time may matter. For example, graduating during a recession may have a large effect on future earnings, see e.g. Oreopoulos et al. (2012). The case with calendar time effects is similar to the case with dynamic treatment effects. Our proposed summary treatment effect parameter involves first computing an average treatment effect for all individuals that are treated in period $t$, and then averaging across all periods. Consider the parameter

$$
\tilde{\theta}_{C}(t)=\sum_{g=2}^{\mathcal{T}} \mathbf{1}\{g \leq t\} A T T(g, t) P(G=g \mid g \leq t) .
$$

Here, $\tilde{\theta}_{C}(t)$ can be interpreted as the average treatment effect for all groups that are treated by period $t$. With $\tilde{\theta}_{C}(t)$ at hand, one can compute

$$
\theta_{C}=\frac{1}{\mathcal{T}-1} \sum_{t=2}^{\mathcal{T}} \tilde{\theta}_{C}(t)
$$


which can be interpreted as the average treatment effect when calendar time matters. When calendar time matters, the most weight is put on groups that are treated in the earliest periods. This is because there are fewer groups available to estimate the average treatment effect in period $t$ when $t$ is small relative to the number of groups available to estimate the average treatment effect in period $t$ when $t$ is large.

Selective Treatment Timing and Dynamic Treatment Effects Finally, we consider the case where the timing of treatment is selected and there are dynamic treatment effects. This might very well be the most relevant case in studying the effect of increasing the minimum wage as $(i)$ states are not likely to raise their minimum wage during a recession and $(i i)$ the effect of the minimum wage takes some time to play out; see e.g. Meer and West (2016).

The fundamental problem with using the dynamic treatment effects approach when there is selective treatment timing is that the composition of the treated group changes when the length of exposure to treatment $(e)$ changes. Without selective treatment timing, this does not matter because when an individual first becomes treated does not affect their outcomes. However, with selective treatment timing, changing the composition of the treatment group can have a big effect (See Figure 1 for an example where the dynamic treatment effect is declining with length of exposure to treatment for all groups but ignoring selective treatment timing leads to the opposite (wrong) conclusion - that the effect of treatment is increasing over time.).

To circumvent such an issue, we consider dynamic treatment effects only for $e \leq e^{\prime}$ and for groups with at least $e^{\prime}$ periods of post-treatment data available. This setup removes the effect of selective treatment timing by keeping the same set of groups across all values of $e$. For example, one could consider the dynamic effect of treatment over three periods by averaging $A T T(g, t)$ for all the groups that have at least three periods of post-treatment observations while not utilizing $A T T(g, t)$ for groups that have less than three periods of post-treatment observations. Note that there is some trade-off here. Setting $e^{\prime}$ small results in many groups satisfying the requirement, but in only being able to study the effect of length of exposure to treatment for relatively few periods. Setting $e^{\prime}$ to be large decreases the number of available groups but allows one to consider 

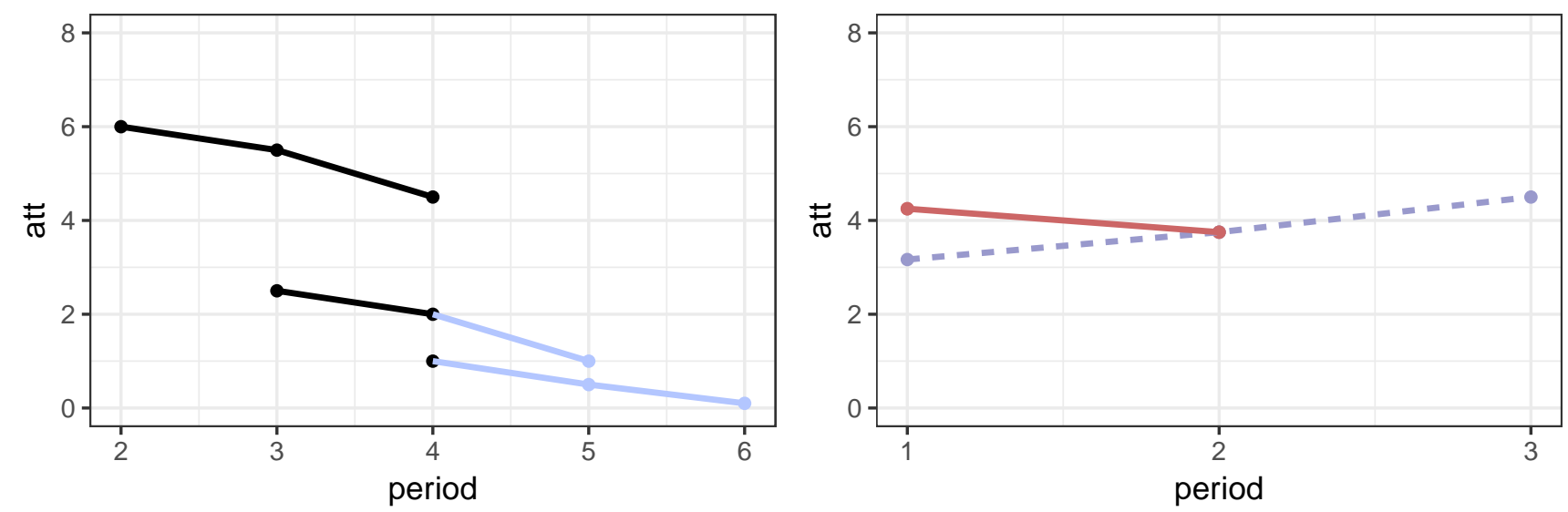

Figure 1: Example of Selective Treatment Timing and Dynamic Treatment Effects

Notes: In this example, there are three groups: G2 (first treated in period 2), G3 (first treated in period 3), and G4 (first treated in period 4). Suppose that the last period available in the sample is period 4; thus, the group-time average treatment effect is available in periods 2 through $4-$ these are the dark lines in the left panel of the figure. The light lines in the left panel represent group-time average treatment effects that are not observed. Each group experiences a declining dynamic treatment effect, but there is also selective treatment timing. Groups that are treated earlier experience larger effects of the treatment. The right panel (dashed line) plots the dynamic treatment effect ignoring selective treatment timing and allowing the composition of the treated group to change. In particular, this means that group G4 is only included in the average for period 1, and group G3 only is included in the average for periods 1 and 2 . In this case, selective treatment timing leads to exactly the wrong interpretation of the dynamic treatment effect it appears as though the effect of the treatment is increasing. The solid line plots the dynamic treatment effect as suggested in Equation (2.6) that adjusts for selective treatment timing and for $e=1,2$ and $e^{\prime}=2$.

the effect of length of exposure to treatment for relatively more periods.

Next, we describe how this proposed summary causal parameter is constructed. Let $\delta_{g t}\left(e, e^{\prime}\right)=$ $1\{t-g+1=e\} 1\left\{T-g+1 \geq e^{\prime}\right\} 1\left\{e \leq e^{\prime}\right\}$. Here, $\delta_{g t}\left(e, e^{\prime}\right)$ is equal to one in the period where group $g$ has been treated for exactly $e$ periods, if group $g$ has at least $e^{\prime}$ post-treatment periods available, and if the length of exposure $e$ is less than the post-treatment periods requirement $e^{\prime}$.

Then, the average treatment effect for groups that have been treated for $e$ periods and have at least $e^{\prime}$ post-treatment periods of data available is given by

$$
\tilde{\theta}_{S D}\left(e, e^{\prime}\right)=\sum_{g=2}^{\mathcal{T}} \sum_{t=2}^{\mathcal{T}} \delta_{g t}\left(e, e^{\prime}\right) \operatorname{ATT}(g, t) P\left(G=g \mid \delta_{g t}\left(e, e^{\prime}\right)=1\right)
$$

which is defined for $e \leq e^{\prime}$. Effectively, we put zero weight on $\operatorname{ATT}(g, t)$ for groups that do not meet the minimum required number of periods in order to prevent the composition of groups from 
changing. Once $\tilde{\theta}_{S D}\left(e, e^{\prime}\right)$ is computed, one can further aggregate it to get

$$
\theta_{S D}\left(e^{\prime}\right)=\frac{1}{\mathcal{T}-e^{\prime}} \sum_{e=1}^{\mathcal{T}-e^{\prime}} \tilde{\theta}_{S D}\left(e, e^{\prime}\right)
$$

which should be interpreted as the average treatment effect for groups with at least $e^{\prime}$ periods of post-treatment data allowing for dynamic treatment effects and selective treatment timing. Such a causal parameter has the strengths of both $\theta_{S}$ and $\theta_{D}$ in (2.4) and (2.5), respectively.

\section{$3 \quad$ Estimation and Inference}

In this section, we study estimation and inference procedures for estimators corresponding to the estimands introduced in Section 2. Note that the nonparametric identification result in Theorem 1 suggests a simple two-step strategy to estimate $A T T(g, t)$. In the first step, estimate the generalized propensity score $p_{g}(x)=P\left(G_{g}=1 \mid X=x, G_{g}+C=1\right)$ for each group $g$, and compute the fitted values for the sample. In the second step, one plugs the fitted values into the sample analogue of $A T T(g, t)$ in (2.1) to obtain estimates of the group-time average treatment effect.

More concisely, we propose to estimate $\operatorname{ATT}(g, t)$ by

$$
\widehat{A T T}(g, t)=\mathbb{E}_{n}\left[\left(\frac{G_{g}}{\mathbb{E}_{n}\left[G_{g}\right]}-\frac{\frac{\hat{p}_{g}(X) C}{1-\hat{p}_{g}(X)}}{\mathbb{E}_{n}\left[\frac{\hat{p}_{g}(X) C}{1-\hat{p}_{g}(X)}\right]}\right)\left(Y_{t}-Y_{g-1}\right)\right],
$$

where $\hat{p}_{g}(\cdot)$ is an estimate of $p_{g}(\cdot)$, and for a generic $Z, \mathbb{E}_{n}[Z]=n^{-1} \sum_{i=1}^{n} Z_{i}$. As noted in Theorem 1, $\operatorname{ATT}(g, t)$ is nonparametrically identified for $2 \leq g \leq t \leq \mathcal{T}$.

With $\widehat{A T T}(g, t)$ in hand, one can use the analogy principle and combine these to estimate the summarized average treatment effect parameters discussed in Section 2.3.

In what follows, we consider the case in which one imposes a parametric restriction on $p_{g}$ and estimates it by maximum likelihood. This is perhaps the most popular approach adopted by practitioners. Nonetheless, under some additional regularity conditions, our results can be extended to allow nonparametric estimators for the $p_{g}(\cdot)$, see e.g. Abadie (2005), Donald and Hsu (2014) and Sant'Anna (2016, 2017). Finally, we note that when propensity score misspecification 
is a concern, one can use the data-driven specification tests proposed by Sant'Anna and Song (2018).

Assumption 5. For all $g=2, \ldots, \mathcal{T},(i)$ there exists a known function $\Lambda: \mathbb{R} \rightarrow[0,1]$ such that $p_{g}(X)=P\left(G_{g}=1 \mid X, G_{g}+C=1\right)=\Lambda\left(X^{\prime} \pi_{g}^{0}\right) ;(i i) \pi_{g}^{0} \in \operatorname{int}(\Pi)$, where $\Pi$ is a compact subset of $\mathbb{R}^{k} ;($ iii $)$ the support of $X, \mathcal{X}$, is a subset of a compact set $S$, and $\mathbb{E}\left[X X^{\prime} \mid G_{g}+C=1\right]$ is positive definite; (iv) let $\mathcal{U}=\left\{x^{\prime} \pi: x \in \mathcal{X}, \pi \in \Pi\right\} ; \forall u \in \mathcal{U}, \exists \varepsilon>0$ such that $\Lambda(u) \in[\varepsilon, 1-\varepsilon], \Lambda(u)$ is strictly increasing and twice continuously differentiable with first derivatives bounded away from zero and infinity, and bounded second derivative; $(v i) \mathbb{E}\left[Y_{t}^{2}\right]<\infty$ for all $t=1, \ldots, \mathcal{T}$.

Assumption 5 is standard in the literature, see e.g. Section 9.2.2 in Amemiya (1985), Example 5.40 in van der Vaart (1998), or Assumption 4.2 in Abadie (2005), and it allows for Logit and Probit models.

Under Assumption 5, $\pi_{g}^{0}$ can be estimated by maximum likelihood:

$$
\hat{\pi}_{g}=\arg \max _{\pi} \sum_{i: G_{i g}+C_{i}=1} G_{i g} \ln \left(p_{g}\left(X_{i}^{\prime} \pi\right)\right)+\left(1-G_{i g}\right) \ln \left(1-p_{g}\left(X_{i}^{\prime} \pi\right)\right)
$$

Let $\mathcal{W}=\left(Y_{1}, \ldots, Y_{\mathcal{T}}, X, G_{1}, \ldots, G_{\mathcal{T}}, C\right)^{\prime}, \hat{p}\left(X_{i}\right)=p_{g}\left(X_{i}^{\prime} \hat{\pi}_{g}\right), \dot{p}_{g}=\partial p_{g}(u) / \partial u, \dot{p}_{g}(X)=$ $\dot{p}_{g}\left(X^{\prime} \pi_{g}^{0}\right)$. Under Assumption $5, \hat{\pi}_{g}$ is asymptotically linear, that is,

$$
\sqrt{n}\left(\hat{\pi}_{g}-\pi_{g}^{0}\right)=\frac{1}{\sqrt{n}} \sum_{i=1}^{n} \xi_{g}^{\pi}\left(\mathcal{W}_{i}\right)+o_{p}(1)
$$

where

$$
\xi_{g}^{\pi}(\mathcal{W})=\mathbb{E}\left[\frac{\left(G_{g}+C\right) \dot{p}_{g}(X)^{2}}{p_{g}(X)\left(1-p_{g}(X)\right)} X X^{\prime}\right]^{-1} X \frac{\left(G_{g}+C\right)\left(G_{g}-p_{g}(X)\right) \dot{p}_{g}(X)}{p_{g}(X)\left(1-p_{g}(X)\right)}
$$

see Lemma A.2 in the Appendix.

\subsection{Asymptotic Theory for Group-Time Average Treatment Effects}

Denote the normalized weights by

$$
w_{g}^{G}=\frac{G_{g}}{\mathbb{E}\left[G_{g}\right]}, \quad w_{g}^{C}=\frac{p_{g}(X) C}{1-p_{g}(X)} / \mathbb{E}\left[\frac{p_{g}(X) C}{1-p_{g}(X)}\right]
$$


and define

$$
\psi_{g t}\left(\mathcal{W}_{i}\right)=\psi_{g t}^{G}\left(\mathcal{W}_{i}\right)-\psi_{g t}^{C}\left(\mathcal{W}_{i}\right)
$$

where

$$
\begin{aligned}
& \psi_{g t}^{G}(\mathcal{W})=w_{g}^{G}\left[\left(Y_{t}-Y_{g-1}\right)-\mathbb{E}\left[w_{g}^{G}\left(Y_{t}-Y_{g-1}\right)\right]\right], \\
& \psi_{g t}^{C}(\mathcal{W})=w_{g}^{C}\left[\left(Y_{t}-Y_{g-1}\right)-\mathbb{E}\left[w_{g}^{C}\left(Y_{t}-Y_{g-1}\right)\right]\right]+M_{g t}^{\prime} \xi_{g}^{\pi}(\mathcal{W}),
\end{aligned}
$$

and

$$
M_{g t}=\frac{\mathbb{E}\left[X\left(\frac{C}{1-p_{g}(X)}\right)^{2} \dot{p}_{g}(X)\left[\left(Y_{i t}-Y_{i g-1}\right)-\mathbb{E}\left[w_{g}^{C}\left(Y_{t}-Y_{g-1}\right)\right]\right]\right]}{\mathbb{E}\left[\frac{p_{g}(X) C}{1-p_{g}(X)}\right]}
$$

which is a $k \times 1$ vector, with $k$ the dimension of $X$, and $\xi_{g}^{\pi}(\mathcal{W})$ is as defined in (3.1).

Finally, let $A T T_{g \leq t}$ and $\widehat{A T T}_{g \leq t}$ denote the vector of $A T T(g, t)$ and $\widehat{A T T}(g, t)$, respectively, for all $g=2, \ldots, \mathcal{T}$ and $t=2, \ldots, \mathcal{T}$ with $g \leq t$. Analogously, let $\Psi_{g \leq t}$ denote the collection of $\psi_{g t}$ across all periods $t$ and groups $g$ such that $g \leq t$.

The next theorem establishes the joint limiting distribution of $\widehat{A T T}_{g \leq t}$.

Theorem 2. Under Assumptions 1-5, for $2 \leq g \leq t \leq \mathcal{T}$,

$$
\sqrt{n}(\widehat{A T T}(g, t)-\operatorname{ATT}(g, t))=\frac{1}{\sqrt{n}} \sum_{i=1}^{n} \psi_{g t}\left(\mathcal{W}_{i}\right)+o_{p}(1)
$$

Furthermore,

$$
\sqrt{n}\left(\widehat{A T T}_{g \leq t}-A T T_{g \leq t}\right) \stackrel{d}{\rightarrow} N(0, \Sigma)
$$

where $\Sigma=E\left[\Psi_{g \leq t}(\mathcal{W}) \Psi_{g \leq t}(\mathcal{W})^{\prime}\right]$

Theorem 2 provides the influence function for estimating the vector of group-time average treatment effects $A T T_{g \leq t}$, as well as its limiting distribution. In order to conduct inference, one can show that the sample analogue of $\Sigma$ is a consistent estimator for $\Sigma$, see e.g. Theorem 4.4 in Abadie (2005) which leads directly to standard errors and pointwise confidence intervals.

Instead of following this route, we propose to use a simple multiplier bootstrap procedure to conduct asymptotically valid inference. Our proposed bootstrap leverages the asymptotic linear 
representations derived in Theorem 2 and inherits important advantages. First, it is easy to implement and very fast to compute. Each bootstrap iteration simply amounts to "perturbing" the influence function by a random weight $V$, and it does not require re-estimating the propensity score in each bootstrap draw. Second, in each bootstrap iteration, there are always observations from each group. This can be a real problem with the traditional empirical bootstrap where there may be no observations from a particular group in some particular bootstrap iteration. Third, computation of simultaneous (in $g$ and $t$ ) valid confidence bands is relatively straightforward. This is particularly important, since researchers are likely to use confidence bands to visualize estimation uncertainty about $A T T(g, t)$. Unlike pointwise confidence bands, simultaneous confidences bands do not suffer from multiple-testing problems, and are guaranteed to cover all $A T T(g, t)$ with at probability at least $1-\alpha$. Finally, we note that our proposed bootstrap procedure can be readily modified to account for clustering, see Remark 2 below.

To proceed, let $\widehat{\Psi}_{g \leq t}(\mathcal{W})$ denote the sample-analogue of $\Psi_{g \leq t}(\mathcal{W})$, where population expectations are replaced by their empirical analogue, and the true generalized propensity score, $p_{g}$, and its derivatives, $\dot{p}_{g}$, are replaced by their MLE estimates, $\hat{p}_{g}$ and $\widehat{\dot{p}}_{g}$, respectively. Let $\left\{V_{i}\right\}_{i=1}^{n}$ be a sequence of iid random variables with zero mean, unit variance and bounded support, independent of the original sample $\left\{\mathcal{W}_{i}\right\}_{i=1}^{n}$. A popular example involves iid Bernoulli variates $\left\{V_{i}\right\}$ with $P(V=1-\kappa)=\kappa / \sqrt{5}$ and $P(V=\kappa)=1-\kappa / \sqrt{5}$, where $\kappa=(\sqrt{5}+1) / 2$, as suggested by Mammen (1993).

We define $\widehat{A T T}_{g \leq t}^{*}$, a bootstrap draw of $\widehat{A T T}_{g \leq t}$, via

$$
\widehat{A T T}_{g \leq t}^{*}=\widehat{A T T}_{g \leq t}+\mathbb{E}_{n}\left[V \cdot \widehat{\Psi}_{g \leq t}(\mathcal{W})\right]
$$

The next theorem establishes the asymptotic validity of the multiplier bootstrap procedure proposed above.

Theorem 3. Under Assumptions 1-5,

$$
\sqrt{n}\left(\widehat{A T T}_{g \leq t}^{*}-\widehat{A T T}_{g \leq t}\right) \underset{*}{\stackrel{d}{\rightarrow}} N(0, \Sigma)
$$

where $\Sigma=\mathbb{E}\left[\Psi_{g \leq t}(\mathcal{W}) \Psi_{g \leq t}(\mathcal{W})^{\prime}\right]$ as in Theorem 2 , and $\underset{*}{\stackrel{d}{\rightarrow}}$ denotes weak convergence (convergence 
in distribution) of the bootstrap law in probability, i.e. conditional on the original sample $\left\{\mathcal{W}_{i}\right\}_{i=1}^{n}$. Additionally, for any continuous functional $\Gamma(\cdot)$

$$
\Gamma\left(\sqrt{n}\left(\widehat{A T T}_{g \leq t}^{*}-\widehat{A T T}_{g \leq t}\right)\right) \underset{*}{\rightarrow} \Gamma(N(0, V)) .
$$

We now describe a practical bootstrap algorithm to compute studentized confidence bands that covers $A T T(g, t)$ simultaneously over all $g \leq t$ with a prespecified probability $1-\alpha$ in large samples. This is similar to the bootstrap procedure used in Kline and Santos (2012), Belloni et al. (2017) and Chernozhukov et al. (2017) in different contexts.

Algorithm 1. 1) Draw a realization of $\left\{V_{i}\right\}_{i=1}^{n}$. 2) Compute $\widehat{A T T}_{g \leq t}^{*}$ as in (3.4), denote its $(g, t)$-element as $\widehat{A T T}^{*}(g, t)$, and form a bootstrap draw of its limiting distribution as $\hat{R}^{*}(g, t)=$ $\sqrt{n}\left(\widehat{A T T}^{*}(g, t)-\widehat{A T T}(g, t)\right)$. 3) Repeat steps 1-2 B times. 4) Compute a bootstrap estimator of the main diagonal of $\Sigma$ such as the bootstrap interquartile range normalized by the interquartile range of the standard normal distribution, $\widehat{\Sigma}(g, t)=\left(q_{0.75}(g, t)-q_{0.25}(g, t)\right) /\left(z_{0.75}-z_{0.25}\right)$, where $q_{p}(g, t)$ is the pth sample quantile of the $\hat{R}^{*}(g, t)$ in the $B$ draws, and $z_{p}$ is the pth quantile of the standard normal distribution. 5) For each bootstrap draw, compute $t-$ test $_{g \leq t}=$ $\max _{(g, t)}\left|\hat{R}^{*}(g, t)\right| \widehat{\Sigma}(g, t)^{-1 / 2}$. 5) Construct $\widehat{c}_{1-\alpha}$ as the empirical $(1-a)$-quantile of the $B$ bootstrap draws of $t$-test $t_{g \leq t}$. 6) Construct the bootstrapped simultaneous confidence band for ATT $(g, t)$, $g \leq t$, as $\widehat{C}(g, t)=\left[\widehat{A T T}(g, t) \pm \widehat{c}_{1-\alpha} \widehat{\Sigma}(g, t)^{-1 / 2} / \sqrt{n}\right]$

The next corollary to Theorem 3 states that the simultaneous confidence band for $A T T(g, t)$ describe in Algorithm 1 has correct asymptotic coverage.

Corollary 1. Under the Assumptions of Theorem 3, for any $0<\alpha<1$, as $n \rightarrow \infty$,

$$
P(A T T(g, t) \in \widehat{C}(g, t): g \leq t) \rightarrow 1-\alpha,
$$

where $\widehat{C}(g, t)$ is as defined in Algorithm 1 .

Remark 2. Frequently, in DID applications, one wishes to account for clustering, see e.g. Bertrand et al. (2004). This is straightforward to implement with the multiplier bootstrap describe above. In the case of county-level minimum wage data, one could allow for clustering at the state level 
by drawing a scalar $U_{s} S$ times - where $S$ is the number of states - and setting $V_{i}=U_{s}$ for all observations $i$ in state $s$, see e.g. Sherman and Le Cessie (2007), Kline and Santos (2012), Cheng et al. (2013), and MacKinnon and Webb (2016, 2017). Such a cluster-robust bootstrap procedure will lead to reliable inference provided that the number of clusters is "large". Finally, it is important to emphasize that our proposed multipliplier-bootstrap procedure accounts for the autocorrelation of the data.

Remark 3. In Algorithm 1 we have required an estimator for the main diagonal of $\Sigma$. However, we note that if one takes $\widehat{\Sigma}(g, t)=1$ for all $(g, t)$, the result in Corollary 1 continue to hold. However, the resulting "constant width" simultaneous confidence band may be of larger length, see e.g. Montiel Olea and Plagborg-Møller (2017) and Freyberger and Rai (2018).

\subsection{Asymptotic Theory for Summary Parameters}

Let $\theta$ generically represent one of the parameters from Section 2.3, including the ones indexed by some variable (for example, $\tilde{\theta}_{S}(g)$ or $\tilde{\theta}_{S D}\left(e, e^{\prime}\right)$ ). Notice that all of the parameters in Section 2.3 can be expressed as weighted averages of $A T T(g, t)$. Write this generically as

$$
\theta=\sum_{g=2}^{\mathcal{T}} \sum_{t=2}^{\mathcal{T}} w_{g t} A T T(g, t)
$$

where $w_{g t}$ are some potentially random weights. $\theta$ can be estimated by

$$
\hat{\theta}=\sum_{g=2}^{\mathcal{T}} \sum_{t=2}^{\mathcal{T}} \widehat{w}_{g t} \widehat{\operatorname{ATT}}(g, t)
$$

where $\widehat{w}_{g t}$ are estimators for $w_{g t}$ such that for all $g, t=2, \ldots, \mathcal{T}$,

$$
\sqrt{n}\left(\widehat{w}_{g t}-w_{g t}\right)=\frac{1}{\sqrt{n}} \sum_{i=1}^{n} \xi_{g t}^{w}\left(\mathcal{W}_{i}\right)+o_{p}(1),
$$

with $\mathbb{E}\left[\xi_{g t}^{w}(\mathcal{W})\right]=0$ and $\mathbb{E}\left[\xi_{g t}^{w}(\mathcal{W}) \xi_{g t}^{w}(\mathcal{W})^{\prime}\right]$ finite and positive definite. Estimators based on the sample analogue of the weights discussed in Section 2.3 satisfy this condition.

Let

$$
l^{w}\left(\mathcal{W}_{i}\right)=\sum_{g=2}^{\mathcal{T}} \sum_{t=2}^{\mathcal{T}} w_{g t} \cdot \psi_{g t}\left(\mathcal{W}_{i}\right)+\xi_{g t}^{w}\left(\mathcal{W}_{i}\right) \cdot \operatorname{ATT}(g, t)
$$


where $\psi_{g t}(\mathcal{W})$ are as defined in (3.3).

The following result follows immediately from Theorem 2, and can be used to conduct asymptotically valid inference for the summary causal parameters $\theta$.

Corollary 2. Under Assumptions 1-5,

$$
\begin{aligned}
\sqrt{n}(\hat{\theta}-\theta) & =\frac{1}{\sqrt{n}} \sum_{i=1}^{n} l^{w}\left(\mathcal{W}_{i}\right)+o_{p}(1) \\
& \stackrel{d}{\rightarrow} N\left(0, \mathbb{E}\left[l^{w}(\mathcal{W})^{2}\right]\right)
\end{aligned}
$$

Corollary 2 implies that one can construct standard errors and confidence intervals for summary treatment effect parameters based on a consistent estimator of $\mathbb{E}\left[l^{w}(\mathcal{W})^{2}\right]$ or by using a bootstrap procedure like the one in Algorithm 1.

\section{Pre-testing the Conditional Parallel Trend Assumption}

So far, we have discussed how one can nonparametrically identify, and conduct asymptotically valid inference about causal treatment effect parameters using conditional DID models with multiple periods and variation in treatment timing. The credibility of our results crucially relies on the conditional parallel trends assumption stated in Assumption 2. This assumption is fundamentally untestable. However, when one imposes a stronger version of the conditional parallel trends assumption, that is, that Assumption 2 holds for all periods $t$, and not only for the periods $g \leq t$, one can assess the reliability of the parallel trends assumption. Relative to Assumption 2, the additional time periods are ones where $g>t$ which are pre-treatment time periods. In this section, we describe how one can construct such a test in our context. Interestingly, our proposed testing procedure exploits more information than simply testing whether $\operatorname{ATT}(g, t)$ are equal to zero for all $2 \leq t<g$, and therefore is able to detect a broader set of violations of the stronger conditional parallel trends condition.

Before proceeding, we state the "augmented" conditional parallel trends assumption that allows us to "pre-test" for the conditional parallel trends assumption stated in Assumption 2. 
Assumption 6 (Augmented Conditional Parallel Trends). For all $t=2, \ldots, \mathcal{T}, g=2, \ldots, \mathcal{T}$,

$$
\mathbb{E}\left[Y_{t}(0)-Y_{t-1}(0) \mid X, G_{g}=1\right]=\mathbb{E}\left[Y_{t}(0)-Y_{t-1}(0) \mid X, C=1\right] \text { a.s.. }
$$

In order to understand how such an assumption leads to testable implications, note that, under Assumption 6 , for $2 \leq t<g \leq \mathcal{T}, \mathbb{E}\left[Y_{t}(0) \mid X, G_{g}=1\right]$ can be expressed as

$$
\begin{aligned}
\mathbb{E}\left[Y_{t}(0) \mid X, G_{g}=1\right] & =\mathbb{E}\left[Y_{t}(0)-Y_{t-1}(0) \mid X, C=1\right]+\mathbb{E}\left[Y_{t-1}(0) \mid X, G_{g}=1\right] \\
& =\mathbb{E}\left[Y_{t}-Y_{t-1} \mid X, C=1\right]+\mathbb{E}\left[Y_{t-1} \mid X, G_{g}=1\right]
\end{aligned}
$$

where the second equality follows since for individuals in group $g$ when $g>t, Y_{t-1}(0)$ is observed since treatment did not started yet. Using exactly the same logic, $Y_{t}(0)$ is also the observed outcome for individuals in group $g$ when $g>t$. Thus, the construction of our test is based on comparing $\mathbb{E}\left[Y_{t}(0) \mid X, G_{g}=1\right]$ in (4.1) to $\mathbb{E}\left[Y_{t} \mid X, G_{g}=1\right]$ for all periods such $2 \leq t<g$ : under Assumption 6 these conditional expectations should be equal.

Formally, the null hypothesis we seek to test is

$$
H_{0}: \mathbb{E}\left[Y_{t}-Y_{t-1} \mid X, G_{g}=1\right]-\mathbb{E}\left[Y_{t}-Y_{t-1} \mid X, C=1\right]=0 \text { a.s. for all } 2 \leq t<g \leq \mathcal{T}
$$

One option to assess $H_{0}$ is to nonparametrically estimate each conditional expectation in (4.2), and compare how close their difference is to zero. Such a procedure would involve choosing smoothing parameters such as bandwidths, assuming additional smoothness conditions of these expectations, potentially ruling out discrete covariates $X$, and would also suffer from the "curse of dimensionality" when the dimension of $X$ is moderate.

In order to avoid these potential drawbacks, one can test an implication of $H_{0}$ by using the results Theorem 1, and compare how close to zero are the estimates of $\operatorname{ATT}(g, t)$ for all $2 \leq t<$ $g \leq \mathcal{T}$. Although intuitive, such a procedure does not exploit all the restrictions imposed by $H_{0}$. For instance, deviations from $H_{0}$ in opposite directions for different values of $X$ could offset each other, implying that one may fail to reject the plausibility of the conditional parallel trends assumption, even when $H_{0}$ is violated in some directions. See Remark 5 at the end of this section for more details about this case. 
We adopt an alternative approach that avoids all the aforementioned drawbacks: it does not involve choosing bandwidths, does not impose additional smoothness conditions, does not suffer from the "curse of dimensionality," and exploits all the testable restrictions implied by the augmented conditional parallel trends assumption. Our proposal builds on the integrated conditional moments (ICM) approach commonly used in the goodness-of-fit literature; see e.g. Bierens (1982), Bierens and Ploberger (1997), Stute (1997), Stinchcombe and White (1998), and Escanciano $(2006 a, b, 2008)$. To the best of our knowledge, we are the first to propose to use ICM to assess the plausibility of the parallel trends assumption, even when there is no treatment timing variation.

Let $w_{g}^{G}$ and $w_{g}^{C}$ be defined as in (3.2). After some algebra, under Assumptions 1-5, we can rewrite $H_{0}$ as

$$
H_{0}: \mathbb{E}\left[\left(w_{g}^{G}-w_{g}^{C}\right)\left(Y_{t}-Y_{t-1}\right) \mid X\right]=0 \text { a.s. for all } 2 \leq t<g \leq \mathcal{T}
$$

see Lemma A.4 in the Appendix. In fact, by exploiting Lemma 1 in Escanciano (2006b), we can further characterize $(4.3)$ as

$$
H_{0}: \mathbb{E}\left[\left(w_{g}^{G}-w_{g}^{C}\right) \gamma(X, u)\left(Y_{t}-Y_{t-1}\right)\right]=0 \forall u \in \Xi \text { for all } 2 \leq t<g \leq \mathcal{T}
$$

where $\Xi$ is a properly chosen space, and the parametric family $\{\gamma(\cdot, u): u \in \Xi\}$ is a family of weighting functions such that the equivalence between (4.3) and (4.4) holds. The most popular weighting functions include $\gamma(X, u)=\exp \left(i X^{\prime} u\right)$ as in Bierens (1982) and $\gamma(X, u)=1\{X \leq u\}$ as in Stute (1997). In the following, to ease the notation, we concentrate our attention to the indicator functions, $\gamma(X, u)=1\{X \leq u\}$, with $\Xi=\mathcal{X}$, the support of the covariates $X$.

The advantage of the representation in (4.4) is that it resembles the expression for $A T T(g, t)$ in (2.1), and therefore we can use a similar estimation procedure that avoids the use of smoothing parameters. To see this, let

$$
J\left(u, g, t, p_{g}\right)=\mathbb{E}\left[\left(w_{g}^{G}-w_{g}^{C}\right) 1(X \leq u)\left(Y_{t}-Y_{t-1}\right)\right]
$$


and, for each $u$ in the support of $X$, we can estimate $J\left(u, g, t, p_{g}\right)$ by

$$
\widehat{J}\left(u, g, t, \hat{p}_{g}\right)=\mathbb{E}_{n}\left[\left(\frac{G_{g}}{\mathbb{E}_{n}\left[G_{g}\right]}-\frac{\frac{\hat{p}_{g}(X) C}{1-\hat{p}_{g}(X)}}{\mathbb{E}_{n}\left[\frac{\hat{p}_{g}(X) C}{1-\hat{p}_{g}(X)}\right]}\right) 1(X \leq u)\left(Y_{t}-Y_{t-1}\right)\right]
$$

where $\hat{p}_{g}$ is a first-step estimator of $p_{g}$.

With $\widehat{J}\left(u, g, t, \hat{p}_{g}\right)$ in hand, one should reject $H_{0}$ when it is not "too close" to zero across different values of $u, g$, and $t, 2 \leq t<g \leq \mathcal{T}$. In order to evaluate the distance from $\widehat{J}\left(u, g, t, \hat{p}_{g}\right)$ to zero, we consider the Cramér-von Mises norm,

$$
C v M_{n}=\int_{\mathcal{X}}\left|\sqrt{n} \widehat{J}_{g>t}(u)\right|_{M}^{2} F_{n, X}(d u)
$$

where $J_{g>t}(u)$ and $\widehat{J}_{g>t}(u)$ denote the vector of $J\left(u, g, t, p_{g}\right)$ and $\hat{J}\left(u, g, t, \hat{p}_{g}\right)$, respectively, for all $g=2, \ldots, \mathcal{T}$ and $t=2, \ldots, \mathcal{T}$, such that $2 \leq t<g \leq \mathcal{T},|A|_{M}$ denotes the weighted seminorm $\sqrt{A^{\prime} M A}$ for a positive semidefinite matrix $M$ and a real vector $A$, and $F_{n, X}$ is the empirical CDF of $X$. To simplify exposition and leverage intuition, we fix $M$ to be a $(\mathcal{T}-1)^{2} \times(\mathcal{T}-1)^{2}$ diagonal matrix such that its $(g, t)$-th diagonal element is given by $1\{g>t\}$. As a result, we can write $C v M_{n}$ as

$$
C v M_{n}=\sum_{g=2}^{\mathcal{T}} \sum_{t=2}^{\mathcal{T}} 1\{g>t\} \int_{\mathcal{X}}\left|\sqrt{n} \widehat{J}\left(u, g, t, \hat{p}_{g}\right)\right|^{2} F_{n, X}(d u) .
$$

This choice of test statistic is similar to the one used by Escanciano (2008) in a different context. However, one can choose some other $M$ or other norms as well.

The key step to derive the asymptotic properties of $C v M_{n}$ is to study the process $\sqrt{n} \widehat{J}\left(u, g, t, \hat{p}_{g}\right)$. Here, note that in contrast to $\widehat{A T T}(g, t), \widehat{J}\left(u, g, t, p_{g}\right)$ is infinite dimensional (since it involves a continuum of $u$ ), and therefore we need to use uniform (instead of pointwise) arguments. Furthermore, we must account for the uncertainty inherited by using the estimated generalized propensity scores $\hat{p}_{g}$ instead of the unknown true $p_{g}$. To accomplish this, we build on the existing literature on empirical processes with a first step estimation of the propensity score; see e.g. Donald and Hsu (2014) and Sant'Anna (2017) for applications in the causal inference context. As before, we focus on the case where the $p_{g}$ is estimated parametrically. 
Define

$$
\psi_{\text {ugt }}^{\text {test }}\left(\mathcal{W}_{i}\right)=\psi_{\text {ugt }}^{G, t e s t}\left(\mathcal{W}_{i}\right)-\psi_{\text {ugt }}^{C, t e s t}\left(\mathcal{W}_{i}\right)
$$

where

$$
\begin{aligned}
& \psi_{\text {ugt }}^{G, \text { test }}(\mathcal{W})=w_{g}^{G}\left[\left(Y_{t}-Y_{t-1}\right) 1(X \leq u)-\mathbb{E}\left[w_{g}^{G} 1(X \leq u)\left(Y_{t}-Y_{t-1}\right)\right]\right], \\
& \psi_{\text {ugt }}^{C, \text { test }}(\mathcal{W})=w_{g}^{C}\left[\left(Y_{t}-Y_{t-1}\right) 1(X \leq u)-\mathbb{E}\left[w_{g}^{C} 1(X \leq u)\left(Y_{t}-Y_{t-1}\right)\right]\right]+M_{u g t}^{\text {test }} \xi_{g}^{\pi}(\mathcal{W}),
\end{aligned}
$$

with $\xi_{g}^{\pi}(\mathcal{W})$ as defined in $(3.1)$, and

$$
M_{u g t}^{\text {test }}=\frac{\mathbb{E}\left[X\left(\frac{C}{1-p_{g}(X)}\right)^{2} \dot{p}_{g}(X)\left[\left(Y_{t}-Y_{t-1}\right) 1(X \leq u)-\mathbb{E}\left[w_{g}^{C} 1(X \leq u)\left(Y_{t}-Y_{t-1}\right)\right]\right]\right]}{\mathbb{E}\left[\frac{p_{g}(X) C}{1-p_{g}(X)}\right]} .
$$

Let $\Psi_{g>t}^{\text {test }}\left(\mathcal{W}_{i} ; u\right)$ denote the vector of $\psi_{u g t}^{\text {test }}\left(\mathcal{W}_{i}\right)$ across all periods $t$ and groups $g$ such that $2 \leq$ $t<g \leq \mathcal{T}$

The next theorem establishes the weak convergence of the process $\sqrt{n} \widehat{J}_{g>t}(u)$ under $H_{0}$, characterizes the limiting null distribution of $C v M_{n}$, and shows that our proposed test is consistent. From these results, we can conclude that our proposed test controls size, and if Assumption 6 does not hold, our test procedure rejects $H_{0}$ with probability approaching one as $n$ goes to infinity. Hence, our tests can indeed be used to assess the reliability of our main identification assumption.

Theorem 4. Suppose Assumptions 1-5 hold. Then,

1. If Assumption 6 holds, i.e., under the null hypothesis (4.4), as $n \rightarrow \infty$,

$$
\sqrt{n} \widehat{J}_{g>t}(u) \Rightarrow \mathbb{G}(u) \text { in } l^{\infty}(\mathcal{X})
$$

where $\Rightarrow$ denote weak convergence in the sense of J. Hoffmann-J申rgensen (see e.g. Definition 1.3.3 in van der Vaart and Wellner (1996)), $\mathcal{X}$ is the support of $X$, and $\mathbb{G}$ is a zero-mean Gaussian process with covariance function

$$
V\left(u_{1}, u_{2}\right)=\mathbb{E}\left[\Psi_{g>t}^{\text {test }}\left(\mathcal{W} ; u_{1}\right) \Psi_{g>t}^{\text {test }}\left(\mathcal{W} ; u_{2}\right)^{\prime}\right]
$$


In particular, as $n \rightarrow \infty$.

$$
C v M_{n} \stackrel{d}{\rightarrow} \int_{\mathcal{X}}|\mathbb{G}(u)|_{M}^{2} F_{X}(d u)
$$

2. If Assumption 6 does not hold, i.e., under the negation of the null hypothesis (4.4)

$$
\lim _{n \rightarrow \infty} P\left(C v M_{n}>c_{\alpha}^{C v M}\right)=1
$$

where $c_{\alpha}^{C v M}=\inf \left\{c \in[0, \infty): \lim _{n \rightarrow \infty} P\left(C v M_{n}>c\right)=\alpha\right\}$.

From Theorem 4, we see that the asymptotic distribution of $C v M_{n}$ depends on the underlying data generating process (DGP) and standardization is complicated. To overcome this problem, we propose to compute critical values with the assistance of the multiplier bootstrap akin to the one discussed in Theorem 3.

To proceed, let $\widehat{\Psi}_{g>t}^{t e s t}(\cdot ; u)$ denote the sample-analogue of $\Psi_{g>t}^{\text {test }}(\cdot ; u)$, where population expectation are replaced by their empirical analogue, and the true generalized propensity score, $p_{g}$, and its derivatives, $\dot{p}_{g}$, are replaced by their MLE estimates, $\hat{p}_{g}$ and $\widehat{\dot{p}}_{g}$, respectively. Let

$$
\widehat{J}_{g>t}^{*}(u)=\mathbb{E}_{n}\left[V \cdot \widehat{\Psi}_{g>t}^{t e s t}(\mathcal{W} ; u)\right]
$$

where $\left\{V_{i}\right\}_{i=1}^{n}$ is defined as in Section 3. The next algorithm provides a step-by-step procedure to approximate $c_{\alpha}$, the critical value of our test $C v M_{n}$.

Algorithm 2. 1) Draw a realization of $\left\{V_{i}\right\}_{i=1}^{n}$. 2) For each $u \in \mathcal{X}$, compute $\widehat{J}_{g>t}^{*}(u)$ as in (4.7). 3) Compute $C v M_{n}^{*}=\int_{\mathcal{X}}\left|\sqrt{n} \widehat{J}_{g>t}^{*}(u)\right|_{M}^{2} F_{n, X}(d u)$. 4) Repeat steps 1-3 B times. 5) Construct $\widehat{c}_{1-\alpha}^{C v M}$ as the empirical $(1-a)$-quantile of the $B$ bootstrap draws of $C v M_{n}^{*}$.

The next Theorem establishes the asymptotic validity of the multiplier bootstrap described in Algorithm 2.

Theorem 5. Suppose Assumptions 1-5 hold. Then, under the null hypothesis (4.4) or under fixed alternatives (i.e., the negation of (4.4)),

$$
\sqrt{n} \widehat{J}_{g>t}^{*}(u) \underset{*}{\Rightarrow} \mathbb{G}(u) \text { in } l^{\infty}(\mathcal{X}),
$$


where $\mathbb{G}(u)$ in $l^{\infty}(\mathcal{X})$ is the same Gaussian process of Theorem 4 and $\underset{*}{\Rightarrow}$ indicates weak convergence in probability under the bootstrap law, see Giné and Zinn (1990). In particular,

$$
C v M_{n}^{*} \stackrel{d}{\rightarrow} \int_{\mathcal{X}}|\mathbb{G}(u)|_{M}^{2} F_{X}(d u)
$$

Remark 4. As discussed in Remark 2, it is straightforward to account for clustering with the multiplier bootstrap described above.

Remark 5. As described above, our proposed test $C v M_{n}$ fully exploits the null hypothesis (4.4), and can detect a broad set of violations against the conditional parallel trends assumption. However, sometimes researchers are also interested in visualizing deviations from the conditional parallel trend assumption, but our proposed Cramér-von Mises test does not directly provide that. In such cases, we note that one can test an implication of the augmented conditional parallel trends assumption, at the cost of losing power against some directions. Namely, under the augmented conditional parallel trends assumptions, $\operatorname{ATT}(g, t)$ should be equal to 0 in periods before individuals become treated, that is, when $g>t$. This test is simple to implement in practice though it is distinct from the tests most commonly employed in DID with multiple periods and multiple groups (see e.g. Autor et al. (2007) and Angrist and Pischke (2008)) which we discuss in more detail in Appendix D in the Supplementary Appendix.

Let $A T T_{g>t}$ denote the "ATT" in periods before an individual in group $g$ is treated (and also satisfying $2 \leq g$ ). Using exactly the same arguments as in Section 3, one can establish the limiting distribution of an estimator of $A T T_{g>t}$ (we omit the details for brevity). And one can implement a test of the augmented parallel trends assumption using a Wald-type test. We also found it helpful in the application to obtain the joint limiting distribution of estimators of $A T T_{g \leq t}$ and $A T T_{g>t}$ (once again using the same arguments as in Section 3) and then reporting uniform confidence bands that cover both pre-tests and estimates of $A T T(g, t)$ across all $g=2, \ldots, \mathcal{T}$ and $t=2, \ldots, \mathcal{T}$. From these uniform confidence bands, one can immediately infer whether or not the implication of the augmented parallel trends assumption is violated. 


\section{The Effect of Minimum Wage Policy on Teen Employ- \\ ment}

In this section, we illustrate the empirical relevance of our proposed methods by studying the effect of the minimum wage on teen employment.

From 1999-2007, the federal minimum wage was flat at $\$ 5.15$ per hour. In July 2007, the federal minimum wage was raised from $\$ 5.15$ to $\$ 5.85$. We focus on county level teen employment in states whose minimum wage was equal to the federal minimum wage at the beginning of the period. Some of these states increased their minimum wage over this period - these become treated groups. Others did not - these are the untreated group. This setup allows us to have more data than local case study approaches. On the other hand, it also allows us to have cleaner identification (state-level minimum wage policy changes) than in studies with more periods; the latter setup is more complicated than ours particularly because of the variation in the federal minimum wage over time. It also allows us to check for internal consistency of identifying assumptions - namely whether or not the identifying assumptions hold in periods before particular states raised their minimum wages.

We use county-level data on teen employment and other county characteristics. County level teen employment as well as minimum wage levels by state comes from Dube et al. (2016) which comes from the Quarterly Workforce Indicators (QWI). See Dube et al. (2016) for a detailed discussion of this dataset. Other county characteristics come from the 2000 County Data Book. These include whether or not a county is located in an MSA, county population in 2000, the fraction of population that are white, educational characteristics from 1990, median income in 1997, and the fraction of population below the poverty level in 1997.

For forty-one states, the federal minimum wage was binding in quarter 2 of 1999 . We omit two states that raised their minimum wage between then and the first quarter of 2004. We drop several other states for lack of data. We use quarterly employment in the first quarter of each year from 2001 to 2007 for employment among teenagers. Alternatively, we could use more periods of data, but this would come at the cost of losing several states due to lack of data. Also, we choose 
first quarter employment because it is further away from the federal minimum wage increase in Q3 of 2007. Our final sample includes county level teen employment for 33 states matched with county characteristics.

Our strategy is to divide the observations based on the timing of when a state increased its minimum wage above the federal minimum wage. States that did not raise their minimum wage during this period form the untreated group. We also have groups of states that increased their minimum wage during 2004, 2006, and 2007. ${ }^{7}$ Before 2004, Illinois did not have a state minimum wage. In Q1 of 2004, Illinois set a state minimum wage of $\$ 5.50$ which was 35 cents higher than the federal minimum wage. In Q1 of 2005, Illinois increased its minimum wage to $\$ 6.50$ where it stayed for the remainder of the period that we consider. No other states changed their minimum wage policy by the first quarter of 2005. In the second quarter of 2005, Florida and Wisconsin set a state minimum wages above the federal minimum wage. In Q3 of 2005, Minnesota also set a state minimum wage. Florida and Wisconsin each gradually increased their minimum wages over time, while Minnesota's was flat over the rest of the period. These three states constitute the treated group for 2006. West Virginia increased its minimum wage in Q3 of 2006; Michigan and Nevada increased their minimum wages in Q4 of 2006; Colorado, Maryland, Missouri, Montana, North Carolina, and Ohio increased their state minimum wages in Q1 of 2007. These states form the 2007 treated group. Among these there is some heterogeneity in the size of the minimum wage increase. For example, North Carolina only increased its minimum wage to $\$ 6.15$ though each state increased its minimum wage to strictly more than the new federal minimum wage of $\$ 5.85$ per hour in Q3 of 2007. At the other extreme, Michigan increased its minimum wage to $\$ 6.95$ and then to $\$ 7.15$ by $\mathrm{Q} 2$ of 2007 .

Figure 2 contains the spatial distribution of state-level minimum wage policy changes in our sample. Dube et al. (2010) argue that differential trends in employment rates across regions bias estimates of the effect of changes in state-level minimum wages. Indeed, Figure 2 shows that states in the Southeast are less likely to increase their minimum wage between 2001 and 2007 than states in the Northeast or Midwest. Table 1 contains the complete details of the exact date when

\footnotetext{
${ }^{7}$ To be precise, we use only employment data from the first quarter of each year. A state is considered to raise its minimum wage in year $y$ if it raised its minimum wage in Q2, Q3, or Q4 of year $y-1$ or in Q1 of year $y$.
} 
a states changed its minimum wage as well as which states are used in our analysis.

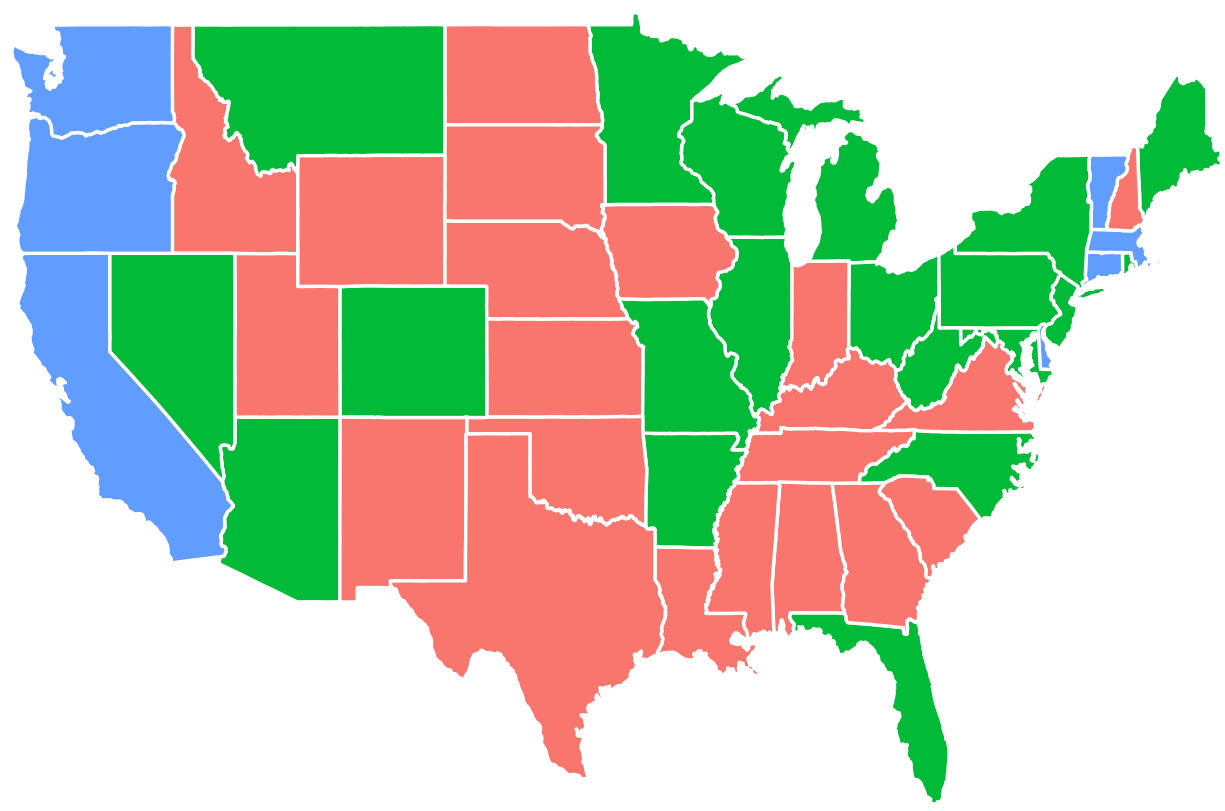

Figure 2: The Spatial Distribution of States by Minimum Wage Policy

Notes: Blue states had minimum wages higher than the federal minimum wage in Q1 of 2000. Green states increased their state minimum wage between Q2 of 2000 and Q1 of 2007. Some of these states are omitted from the main dataset either due to missing data or being located in the Northern census region where there are no states that did not raise their minimum wage between 2000 and 2007 with available data. Otherwise, the green states constitute the treated group. See Table 1 for exact timing of each state's change in the minimum wage. Red states did not increase their minimum wage over the period from 2000 to 2007.

Summary statistics for county characteristics are provided in Table 2. As discussed above, treated counties are much more likely to be in the South. They also have much lower population (on average 53,000 compared to 94,000 for treated counties). The proportion of black residents is much higher in treated counties (on average, $10 \%$ compared to $6 \%$ for untreated counties). There are smaller differences in the fraction with high school degrees and the poverty rate though the differences are both statistically significant. Treated counties have a somewhat smaller fraction of high school graduates and a somewhat higher poverty rate.

In the following we discuss different set of results using different identification strategies. In particular, we consider the cases in which one would assume that the parallel trends assumption 
Table 1: Timing of States Raising Minimum Wage

\begin{tabular}{|c|c|c|c|}
\hline State & Year-Quarter Raised MW & State & Year-Quarter Raised MW \\
\hline Alabama & Never Increased & Montana* & $2007-1$ \\
\hline Alaska & Always Above & Nebraska* & Never Increased \\
\hline Arizona & $2007-1$ & Nevada* & $2006-4$ \\
\hline Arkansas & $2006-4$ & New Hampshire & Never Increased \\
\hline California & Always Above & New Jersey & $2005-4$ \\
\hline Colorado* & $2007-1$ & New Mexico* & Never Increased \\
\hline Connecticut & Always Above & New York & $2005-1$ \\
\hline Delaware & 1999-2 & North Carolina* & $2007-1$ \\
\hline Florida* & $2005-2$ & North Dakota* & Never Increased \\
\hline Georgia* & Never Increased & Ohio* & $2007-1$ \\
\hline Hawaii & Always Above & Oklahoma* & Never Increased \\
\hline Idaho* & Never Increased & Oregon & Always Above \\
\hline Illinois* & $2004-1$ & Pennsylvania & $2007-1$ \\
\hline Indiana* & Never Increased & Rhode Island & 1999-3 \\
\hline Iowa* & $2007-2$ & South Carolina* & Never Increased \\
\hline Kansas* & Never Increased & South Dakota* & Never Increased \\
\hline Kentucky & Never Increased & Tennessee* & Never Increased \\
\hline Louisiana* & Never Increased & Texas* & Never Increased \\
\hline Maine & $2002-1$ & Utah* $^{*}$ & Never Increased \\
\hline Maryland* & $2007-1$ & Vermont & Always Above \\
\hline Massachusetts & Always Above & Virginia* & Never Increased \\
\hline Michigan* & $2006-4$ & Washington & 1999-1 \\
\hline Minnesota* & $2005-3$ & West Virginia* & $2006-3$ \\
\hline Mississippi & Never Increased & Wisconsin* & $2005-2$ \\
\hline Missouri* & $2007-1$ & Wyoming & Never Increased \\
\hline
\end{tabular}

Notes: The timing of states increasing their minimum wage above the federal minimum wage of $\$ 5.15$ per hour which was set in Q4 of 1997 and did not change again until it increased in Q3 of 2007. States that are ultimately included in the main sample are denoted with a ${ }^{*}$. States that had minimum wages higher than the federal minimum wage at the beginning of the period are excluded. We also exclude some states who raised their minimum wage very soon after the federal minimum wage increase, some others due to lack of data availability, and those in the Northern Census region. There are 29 states ultimately included in the sample. 
Table 2: Summary Statistics for Main Dataset

\begin{tabular}{lcccc}
\hline & Treated States & Untreated States & Diff & P-val on Difference \\
\hline Midwest & 0.59 & 0.34 & 0.259 & 0.00 \\
South & 0.27 & 0.59 & -0.326 & 0.00 \\
West & 0.14 & 0.07 & 0.067 & 0.00 \\
Black & 0.06 & 0.10 & -0.042 & 0.00 \\
HS Graduates & 0.59 & 0.55 & 0.327 & 0.00 \\
Population (1000s) & 94.32 & 53.43 & 40.896 & 0.00 \\
Poverty Rate & 0.13 & 0.16 & -0.259 & 0.00 \\
\hline
\end{tabular}

Notes: Summary statistics for counties located in states that raised their minimum wage between Q2 of 2003 and Q1 of 2007 (treated) and states whose minimum wage was effectively set at the federal minimum wage for the entire period (untreated). The sample consists of 2284 counties.

Sources: Quarterly Workforce Indicators and 2000 County Data Book

would hold unconditionally, and when it holds only after controlling for observed characteristics $X$.

The first set of results come from using the unconditional parallel trends assumption to estimate the effect of raising the minimum wage on teen employment. The results for group-time average treatment effects are reported in Figure 3 along with a uniform 95\% confidence band. All inference procedures use clustered bootstrapped standard errors at the county level, and account for the autocorrelation of the data. The plot contains pre-treatment estimates that can be used to test the parallel trends assumption as well as treatment effect estimates in post-treatment periods.

The group-time average treatment effect estimates provide fairly strong support for state-level policies that increased the minimum wage leading to a reduction in teen employment. For 4 out of 7 group-time average treatment effects, there is a clear statistically significant negative effect on employment. The other three are marginally insignificant (and negative). The group-time average treatment effects range from $2.3 \%$ lower teen employment to $13.6 \%$ lower teen employment. The simple average (weighted only by group size) is $5.2 \%$ lower teen employment (see Table 3). A two-way fixed effects model with a post treatment dummy variable also provides similar results, indicating 3.7\% lower teen employment due to increasing the minimum wage. In light of the literature on the minimum wage these results are not surprising as they correspond to the types of regressions that tend to find that increasing the minimum wage decreases employment; see the 
discussion in Dube et al. (2010).

States that raised minimum wage in 2004

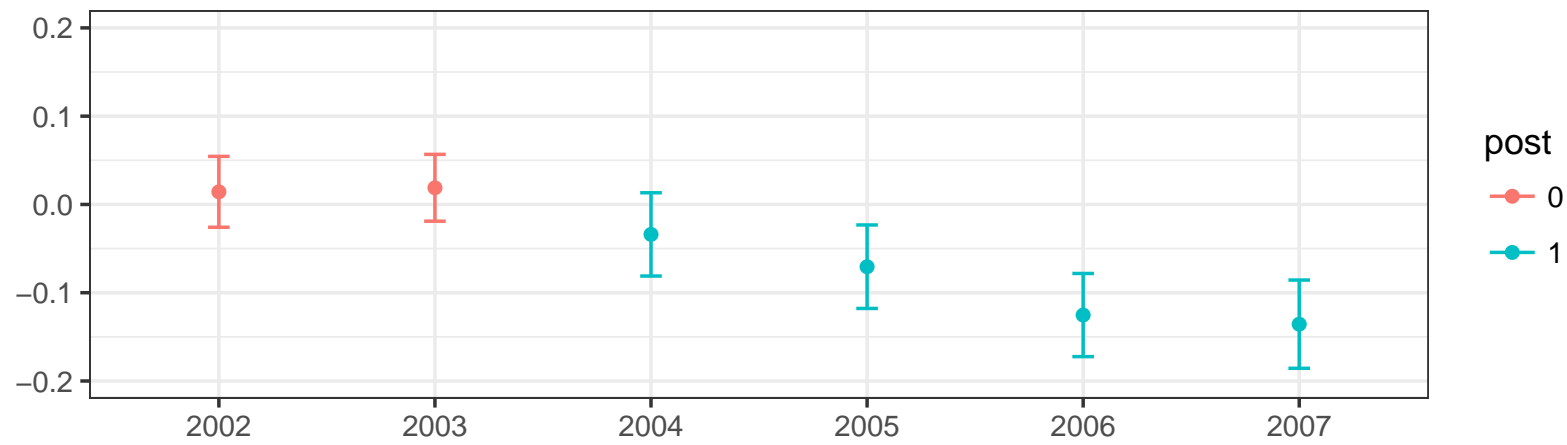

States that raised minimum wage in 2006

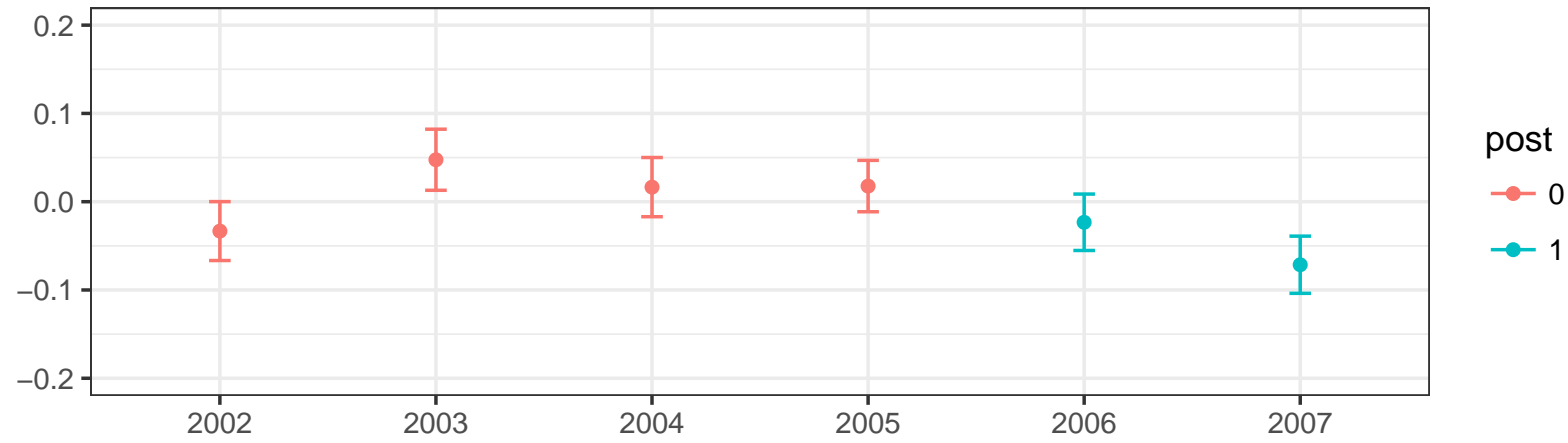

States that raised minimum wage in 2007

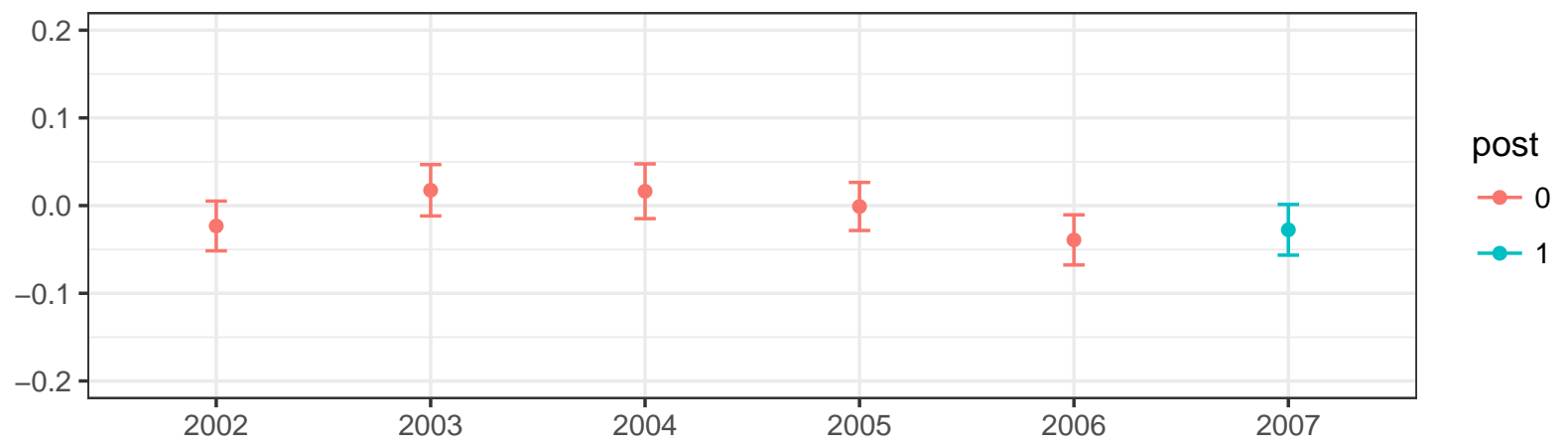

Figure 3: Minimum Wage Results under Unconditional DID

Notes: The effect of the minimum wage on teen employment estimated under the Unconditional DID Assumption. Red lines give point estimates and uniform 95\% confidence bands for pretreatment periods allowing for clustering at the county level. Under the null hypothesis of the Conditional DID Assumption holding in all periods, these should be equal to 0. Blue lines provide point estimates and uniform $95 \%$ confidence bands for the treatment effect of increasing the minimum wage allowing for clustering at the county level. The top panel includes states that increased their minimum wage in 2004, the middle panel includes states that increased their minimum wage in 2006, and the bottom panel includes states that increased their minimum wage in 2007. No states raised their minimum wages in other years prior to 2007. 
As in Meer and West (2016), there also appears to be a dynamic effect of increasing the minimum wage. For Illinois (the only state in the group that first raised its minimum wage in 2004), teen employment is $3.4 \%$ lower on average in 2004 than it would have been if the minimum wage had not been increased. In 2005, teen employment is estimated to $7.1 \%$ lower; in $2006,12.5 \%$ lower; and in 2007, 13.6\% lower. For states first treated in 2006, there is a small effect in 2006 $2.3 \%$ lower teen employment; however, it is larger in $2007-7.1 \%$ lower teen employment.

Table 3 reports aggregated treatment effect measures. Allowing for dynamic treatment effects is perhaps the most useful for our study. These parameters paint largely the same picture as the group-time average treatment effects. The effect of increasing the minimum wage on teen employment appears to be negative and getting stronger the longer states are exposed to the higher minimum wage. In particular, in the first year that a state increases its minimum wage, teen employment is estimated to decrease by $2.7 \%$, in the second year it is estimated to decrease by $7.1 \%$, in the third year by $12.5 \%$, and in the fourth year by $13.6 \%$. Notice that the last two dynamic treatment effect estimates are exactly the same as the estimates coming from Illinois alone because Illinois is the only state that is treated for more than two years. These results are robust to keeping the treated group constant to make sure that selective treatment timing does not bias the results (see the row in Table 3 labeled 'Selectivity and Dynamics'). When we restrict the sample to only include groups with at least two years of exposure to treatment (and only considering the first two periods of exposure which keeps the groups constant across length of exposure), we estimate that the effect of minimum wage increases in the first period of exposure is $2.7 \%$ lower teen employment and $7.1 \%$ lower teen employment in the second period. ${ }^{8}$

Allowing for calendar time effects or selective treatment timing also is consistent with the idea that states that increased their minimum wage experienced negative effects on teen employment relative to what they would have experienced if they had not increased their minimum wage.

We consider testing the unconditional parallel trends assumption. First, since the confidence

\footnotetext{
${ }^{8}$ Notice that these estimates are exactly the same as in the first two periods for the dynamic treatment effect estimates that do not condition on the group remaining constant. The reason that they are the same for the first period is coincidental; the estimated effect of the minimum wage in 2007 for the group of states first treated in 2007 is $2.76 \%$ lower teen employment which just happens to correspond to the estimated effect in the latter case. For the second period, they correspond by construction.
} 
Table 3: Aggregate Treatment Effect Parameters under Unconditional Parallel Trends

Standard DID

Partially Aggregated

Single Parameters

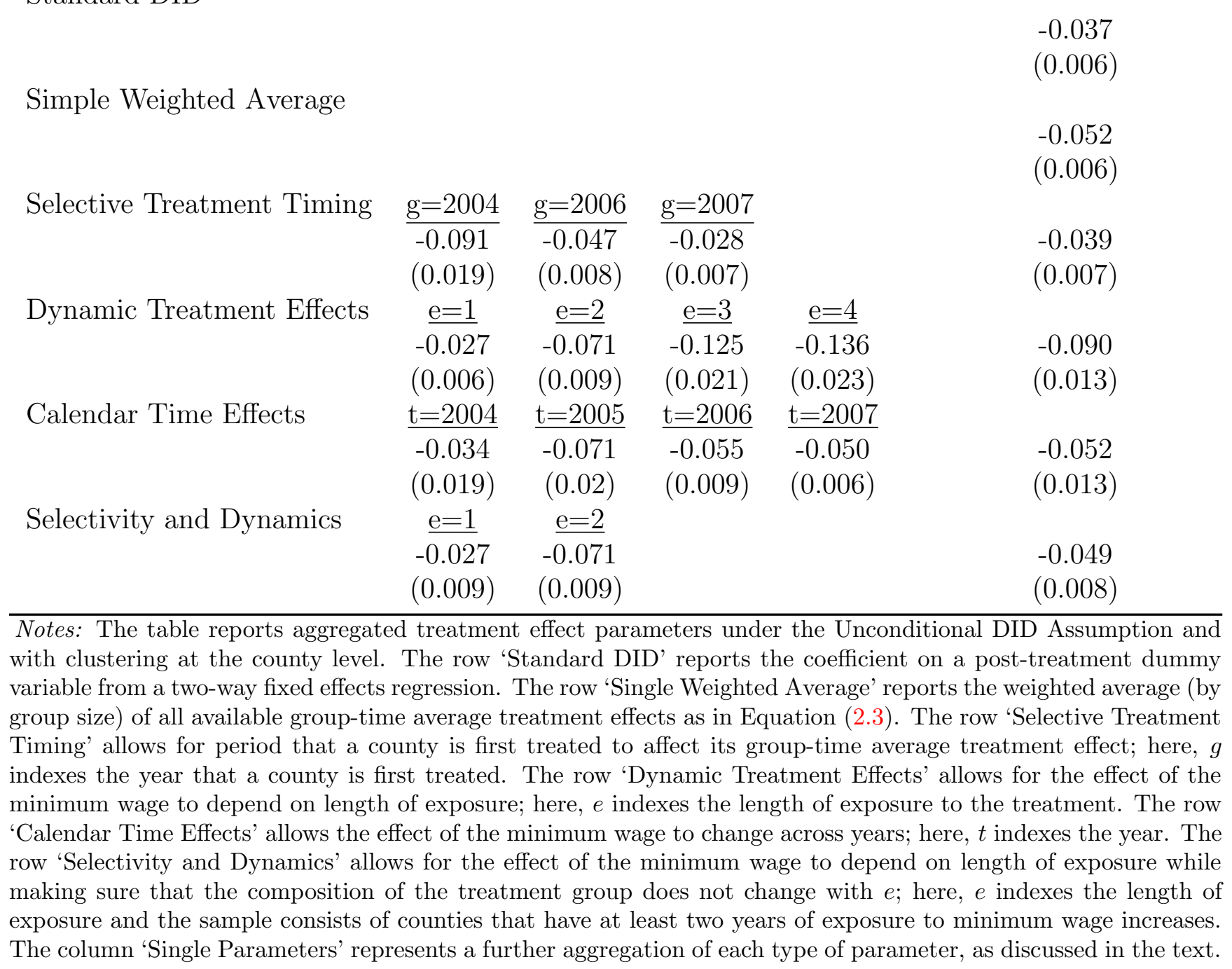

bands in Figure 3 are uniform, one can immediately infer that the unconditional parallel trends assumption should be rejected based on the implication of the unconditional parallel trends assumption that the "ATT" in periods before treatment should be equal to 0. Likewise, our proposed test also rejects the unconditional parallel trends assumption (p-value: 0.000$)$. The estimated uniform confidence bands in Figure 3 also provide some insight into how to think about our pre-tests. For the group first treated in 2004, the parallel trends assumption is not rejected in any period. For the group first treated in 2006, it is rejected in 2003; for the group first treated in 2007, it is rejected in 2006. Interestingly, with the exception of 2006 for the group first treated in 2007, in 
each of the cases where it is rejected, the placebo estimates are positive.

The second set of results come from using the conditional parallel trends assumption; that is, we assume only that counties with the same characteristics would follow the same trend in teen employment in the absence of treatment. The county characteristics that we use are region of the country, county population, county median income, the fraction of the population that is white, the fraction of the population with a high school education, and the county's poverty rate. Estimation requires a first step estimation of the generalized propensity score. For each generalized propensity score, we estimate a logit model that includes each county characteristic along with quadratic terms for population and median income ${ }^{9}$. In particular, the conditional results allow for differential trends in teen employment across different regions as well as in the other county characteristics mentioned above. In what follows, all inference procedures use clustered bootstrapped standard errors at the county level.

For comparison's sake, we first estimate the coefficient on a post-treatment dummy variable in a model with individual fixed effects and region-year fixed effects. This is very similar to one of the sorts of models that Dube et al. (2010) finds to eliminate the correlation between the minimum wage and employment. Like Dube et al. (2010), using this specification, we find that the estimated coefficient is small and not statistically different from 0 . However, one must have in mind that the approach we proposed in this article is different from the two-way fixed effects regression. In particular, we explicitly identify group-time average treatment effects for different groups and different times, allowing for arbitrary treatment effect heterogeneity as long as the conditional parallel trends assumption is satisfied. Thus, our causal parameters have a clear interpretation. As pointed out by Wooldridge (2005), Chernozhukov et al. (2013), de Chaisemartin and D'Haultfoeuille (2016), Borusyak and Jaravel (2017), Goodman-Bacon (2017) and Słoczyński (2017), the same may not be true for two-way fixed effect regressions in the presence of treatment effect heterogeneity. ${ }^{10}$

\footnotetext{
${ }^{9}$ Using the propensity score specification tests proposed by Sant'Anna and Song (2018), we fail to reject the null hypothesis that these models are correctly specified at the usual significance levels.

${ }^{10}$ Our approach is also different from that of Dube et al. (2010) in several other ways that are worth mentioning. We focus on teen employment; Dube et al. (2010) considers employment in the restaurant industry. Their most similar specification to the one mentioned above includes census division-time fixed effects rather than region-time fixed effects though the results are similar. Finally, our period of analysis is different from theirs; in particular,
} 
States that raised minimum wage in 2004

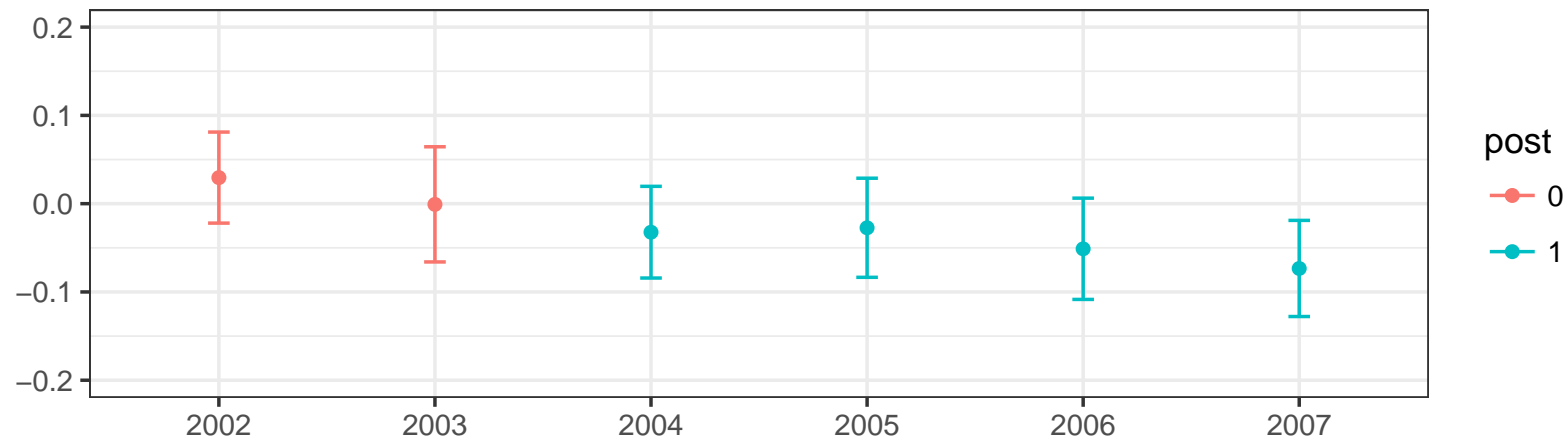

States that raised minimum wage in 2006

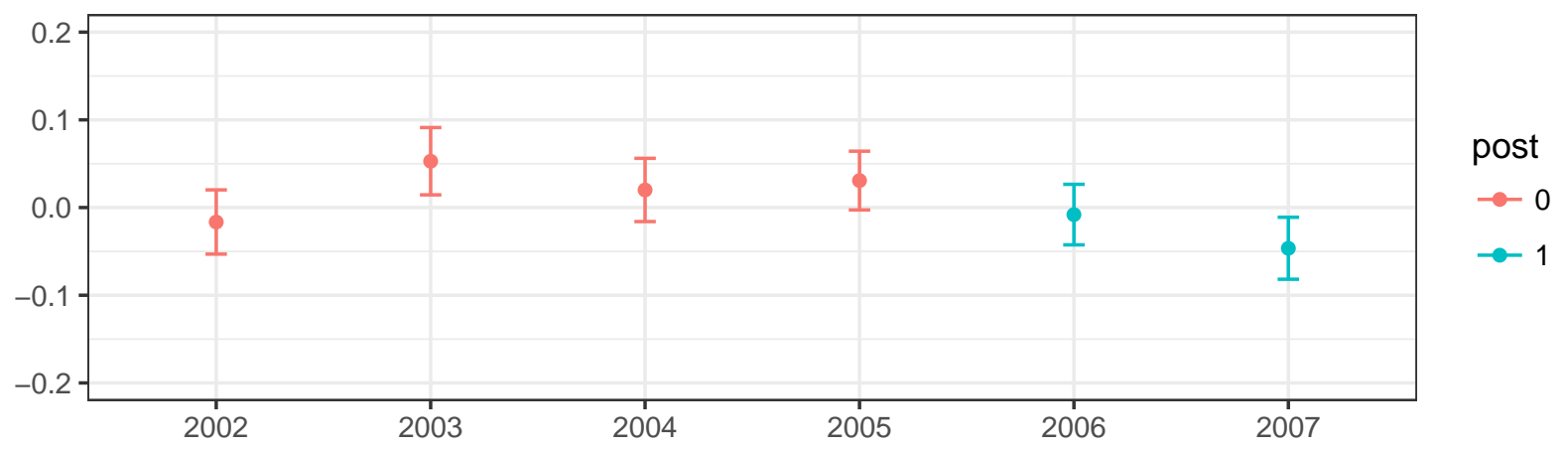

States that raised minimum wage in 2007

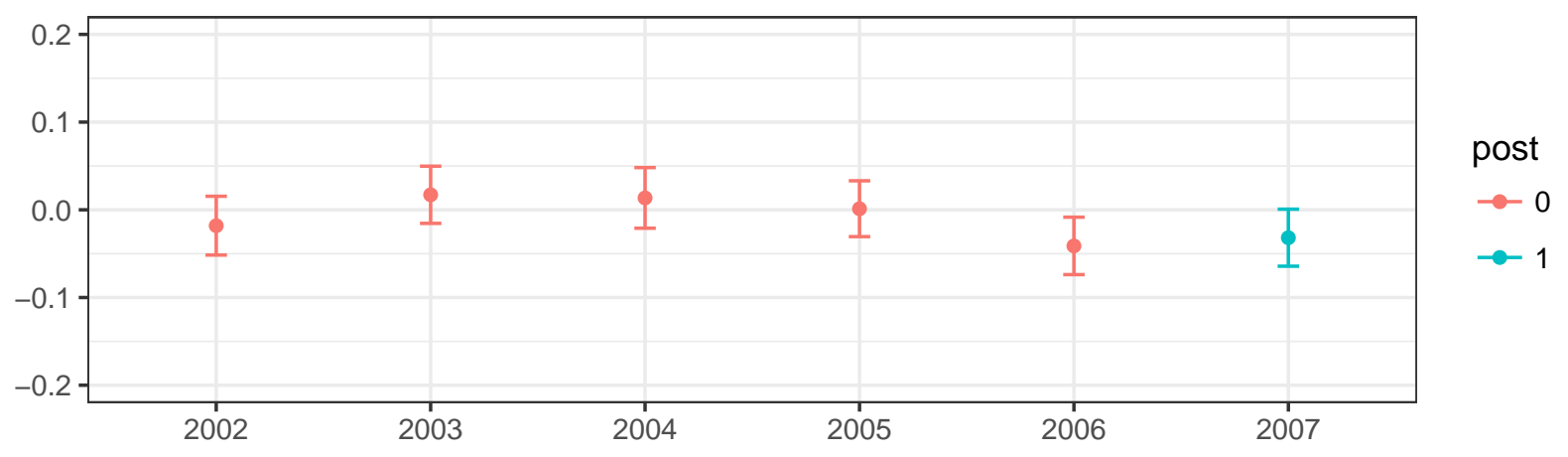

Figure 4: Minimum Wage Results under Conditional DID

Notes: The effect of the minimum wage on teen employment estimated under the Conditional DID Assumption. Red lines give point estimates and uniform 95\% confidence bands for pretreatment periods allowing for clustering at the county level. Under the null hypothesis of the Conditional DID Assumption holding in all periods, these should be equal to 0. Blue lines provide point estimates and uniform $95 \%$ confidence bands for the treatment effect of increasing the minimum wage allowing for clustering at the county level. The top panel includes states that increased their minimum wage in 2004, the middle panel includes states that increased their minimum wage in 2006, and the bottom panel includes states that increased their minimum wage in 2007. No states raised their minimum wages in other years prior to 2007.

there are no federal minimum wage changes over the periods we analyze. 
The results using our approach are available in Figure 4 and Table 4. Interestingly, we find quite different results using our approach than are suggested by the two-way fixed effect regression approach. In particular, we continue to find evidence that increasing the minimum wage tended to reduce teen employment. The estimated group-time average treatment effects range from $0.8 \%$ lower teen employment (not statistically different from 0) in 2006 for the group of states first treated in 2006 to $7.3 \%$ lower teen employment in 2007 for states first treated in 2004 . Now only 2 of 7 group-time average treatment effects are statistically significant. The pattern of dynamic treatment effects where the effect of minimum wage increases tends to increase with length of exposure is the same as in the unconditional case. Similarly, using our aggregated treatment effect parameters, allowing for dynamic treatment effects, we estimate that increasing the minimum wage led on average to $4.8 \%$ lower teen employment. Allowing for dynamic treatment effects and selective treatment timing, we estimate that increasing the minimum wage lowers teen employment by $2.8 \%$.

The evidence of the negative effect of minimum wage increases is somewhat mitigated by the fact that we reject the conditional parallel trends assumption in pre-treatment periods. This is immediately evident from Figure 4 because we can reject that the "ATT" is equal to 0 in 2 out of 11 pre-treatment periods. Using the consistent Cramér-von Mises tests discussed in Section 4, we also reject the conditional parallel trends assumption (p-value: 0.000).

Overall, our results suggests that the minimum wage decreased teen employment in states that increased their minimum wage relative to what it would have been had those states not increased their minimum wage. Nonetheless, our proposed tests indicate that the parallel trends assumption should be rejected in pre-treatment periods, implying that the DID research design may lead to non-reliable conclusions. Perhaps not surprisingly, given the amount of disagreement in the minimum wage literature, our results should be interpreted with care and are ultimately inconclusive. 
Table 4: Aggregate Treatment Effect Parameters under Conditional Parallel Trends

Standard DID

Partially Aggregated

Single Parameters

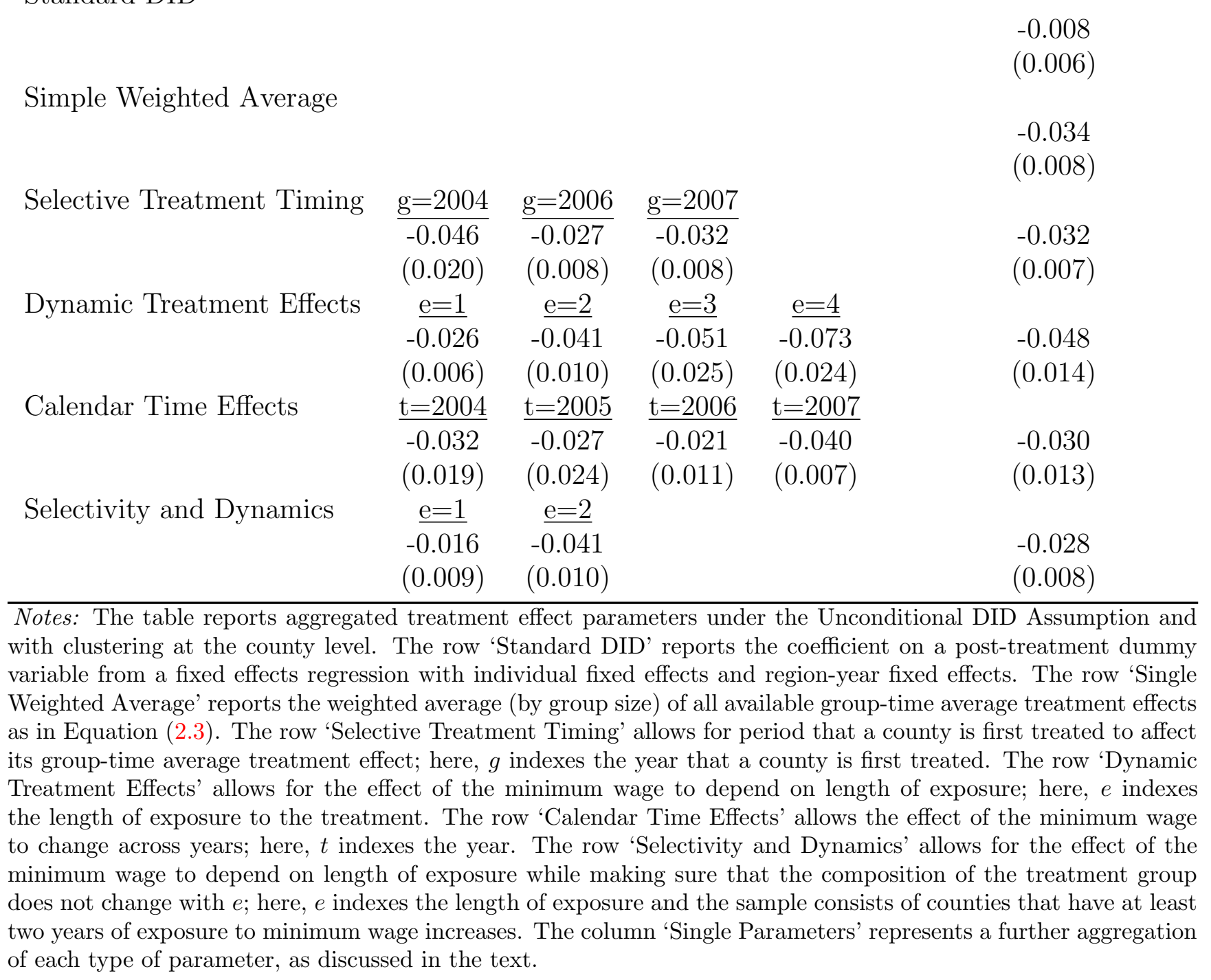

\section{Conclusion}

This paper has considered Difference-in-Differences methods in the case where there are more than two periods and individuals can become treated at different points in time - a commonly encountered setup in empirical work in economics. In this setup, we have suggested computing group-time average treatment effects, $\operatorname{ATT}(g, t)$, that are the average treatment effect in period $t$ for the group of individuals first treated in period $g$. Unlike the more common approach of running a regression with a post-treatment dummy variable, $A T T(g, t)$ corresponds to a well 
defined treatment effect parameter. And once $A T T(g, t)$ has been obtained for different values of $g$ and $t$, they can be aggregated into a single parameter, though the exact implementation depends on the particular case. We view such a flexibility as a plus of our proposed methodology.

Given that our nonparametric identification results are constructive, we proposed to estimate $A T T(g, t)$ using its sample analogue. We established consistency and asymptotically normality of the proposed estimators, and proved the validity of a powerful, but easy to implement, multiplier bootstrap procedure to construct simultaneous confidence bands for $\operatorname{ATT}(g, t)$. Importantly,

we have also proposed a new pre-test for the the reliability of the conditional parallel trends assumption.

We applied our approach to study the effect of minimum wage increases on teen employment. We found some evidence that increasing the minimum wage led to reductions in teen employment and found strikingly different results from the more common approach of interpreting the coefficient on a post-treatment dummy variable as the effect of the minimum wage on employment. However, using the pre-tests developed in the current paper, we found evidence against both the unconditional and conditional parallel trends assumption.

\section{Appendix A: Mathematical Proofs}

We provide the proofs of our results in this appendix. Before proceeding, we first state and prove several auxiliary lemmas that help us proving our main theorems.

Let

$$
A T T_{X}(g, t)=\mathbb{E}\left[Y_{t}(1)-Y_{t}(0) \mid X, G_{g}=1\right]
$$

Lemma A.1. Under Assumptions 1-4, and for $2 \leq g \leq t \leq \mathcal{T}$,

$$
A T T_{X}(g, t)=\mathbb{E}\left[Y_{t}-Y_{g-1} \mid X, G_{g}=1\right]-\mathbb{E}\left[Y_{t}-Y_{g-1} \mid X, C=1\right] \text { a.s.. }
$$

Proof of Lemma A.1: In what follows, take all equalities to hold almost surely (a.s.). Notice that for identifying $A T T_{X}(g, t)$, the key term is $E\left[Y_{t}(0) \mid X, G_{g}=1\right]$. And notice that for $h>s$, $E\left[Y_{s}(0) \mid X, G_{s}=1\right]=E\left[Y_{s} \mid X, G_{h}=1\right]$, which holds because in time periods before an individual 
is first treated, their untreated potential outcomes are observed outcomes. Also, note that, for $2 \leq g \leq t \leq \mathcal{T}$

$$
\begin{aligned}
\mathbb{E}\left[Y_{t}(0) \mid X, G_{g}=1\right] & =\mathbb{E}\left[\Delta Y_{t}(0) \mid X, G_{g}=1\right]+\mathbb{E}\left[Y_{t-1}(0) \mid X, G_{g}=1\right] \\
& =\mathbb{E}\left[\Delta Y_{t} \mid X, C=1\right]+\mathbb{E}\left[Y_{t-1}(0) \mid X, G_{g}=1\right]
\end{aligned}
$$

where the first equality holds by adding and subtracting $E\left[Y_{t-1}(0) \mid X, G_{g}=1\right]$ and the second equality holds by Assumption 2. If $g=t-1$, then the last term in the final equation is identified; otherwise, one can continue recursively in similar way to (A.1) but starting with $\mathbb{E}\left[Y_{t-1}(0) \mid X, G_{g}=\right.$ 1]. As a result,

$$
\begin{aligned}
\mathbb{E}\left[Y_{t}(0) \mid X, G_{g}=1\right] & =\sum_{j=0}^{t-g} \mathbb{E}\left[\Delta Y_{t-j} \mid X, C=1\right]+\mathbb{E}\left[Y_{g-1} \mid X, G_{g}=1\right] \\
& =\mathbb{E}\left[Y_{t}-Y_{g-1} \mid X, C=1\right]+\mathbb{E}\left[Y_{g-1} \mid X, G_{g}=1\right]
\end{aligned}
$$

Combining (A.2) with the fact that, for all $g \leq t, \mathbb{E}\left[Y_{t}(1) \mid X, G_{g}=1\right]=\mathbb{E}\left[Y_{t} \mid X, G_{g}=1\right]$ (which holds because observed outcomes for group $g$ in period $t$ with $g \leq t$ are treated potential outcomes), implies the result.

Next, recall that

$$
\hat{\pi}_{g}=\arg \max _{\pi} \sum_{i: G_{i g}+C_{i}=1} G_{i g} \ln \left(p_{g}\left(X_{i}^{\prime} \pi\right)\right)+\left(1-G_{i g}\right) \ln \left(1-p_{g}\left(X_{i}^{\prime} \pi\right)\right)
$$

$\dot{p}_{g}=\partial p_{g}(u) / \partial u, \dot{p}_{g}(X)=\dot{p}_{g}\left(X^{\prime} \pi_{g}^{0}\right)$, and $\pi_{g}^{0}$ is the true, unknown vector of parameter indexed the generalized propensity score $p_{g}(X)=\mathbb{E}\left[G_{g}=1 \mid X, G_{g}+C=1\right]$.

Lemma A.2. Under Assumption 5,

$$
\sqrt{n}\left(\hat{\pi}_{g}-\pi_{g}^{0}\right)=\frac{1}{\sqrt{n}} \sum_{i=1}^{n} \xi_{g}^{\pi}\left(\mathcal{W}_{i}\right)+o_{p}(1)
$$

where

$$
\xi_{g}^{\pi}(\mathcal{W})=\mathbb{E}\left[\frac{\left(G_{g}+C\right) \dot{p}_{g}(X)^{2}}{p_{g}(X)\left(1-p_{g}(X)\right)} X X^{\prime}\right]^{-1} X \frac{\left(G_{g}+C\right)\left(G_{g}-p_{g}(X)\right) \dot{p}_{g}(X)}{p_{g}(X)\left(1-p_{g}(X)\right)}
$$


Proof of Lemma A.2: Let $n_{g c}=\sum_{i=1}^{n}\left(C_{i}+G_{i g}\right)$. Under Assumption 5, from Theorem 5.39 and Example 5.40 in van der Vaart (1998), we have

$$
\begin{aligned}
& \sqrt{n_{g c}}\left(\hat{\pi}_{g}-\pi_{g}^{0}\right) \\
& =\frac{1}{\sqrt{n_{g c}}} \sum_{i: G_{i g}+C_{i}=1}\left(\mathbb{E}\left[\frac{\dot{p}_{g}(X)^{2}}{p_{g}(X)\left(1-p_{g}(X)\right)} X X^{\prime} \mid G_{g}+C=1\right]^{-1} X_{i} \frac{\left(G_{i g}-p_{g}\left(X_{i}\right)\right) \dot{p}_{g}\left(X_{i}\right)}{p_{g}\left(X_{i}\right)\left(1-p_{g}\left(X_{i}\right)\right)}+o_{p}(1)\right. \\
& =\frac{\mathbb{E}\left[G_{g}+C\right]}{\sqrt{n_{g c}}} \sum_{i=1}^{n}\left(\mathbb{E}\left[\frac{\left(G_{g}+C\right) \dot{p}_{g}(X)^{2}}{p_{g}(X)\left(1-p_{g}(X)\right)} X X^{\prime}\right]^{-1} X_{i} \frac{\left(G_{i g}+C_{i}\right)\left(G_{i g}-p_{g}\left(X_{i}\right)\right) \dot{p}_{g}\left(X_{i}\right)}{p_{g}\left(X_{i}\right)\left(1-p_{g}\left(X_{i}\right)\right)}\right)+o_{p}(1) \\
& =\frac{\mathbb{E}_{n}\left[G_{g}+C\right]}{\sqrt{n_{g c}}} \sum_{i=1}^{n} \xi_{g}^{\pi}\left(\mathcal{W}_{i}\right)+o_{p}(1) \\
& =\frac{\sqrt{n_{g c}}}{n} \sum_{i=1}^{n} \xi_{g}^{\pi}\left(\mathcal{W}_{i}\right)+o_{p}(1) .
\end{aligned}
$$

Thus,

$$
\begin{aligned}
\sqrt{n}\left(\hat{\pi}_{g}-\pi_{g}^{0}\right) & =\frac{\sqrt{n}}{\sqrt{n_{g c}}} \sqrt{n_{g c}}\left(\hat{\pi}_{g}-\pi_{g}^{0}\right) \\
& =\frac{1}{\sqrt{n}} \sum_{i=1}^{n} \xi_{g}^{\pi}\left(\mathcal{W}_{i}\right)+o_{p}(1),
\end{aligned}
$$

and the proof is complete.

For an arbitrary $\pi$, let $p_{g}(x ; \pi)=p_{g}\left(x^{\prime} \pi\right), \dot{p}_{g}(x ; \pi)=\dot{p}_{g}\left(x^{\prime} \pi\right)$, for all $g=2, \ldots, \mathcal{T}$. Define the classes of functions,

$$
\begin{aligned}
& \mathcal{H}_{1, g}=\left\{(x, c) \mapsto c \frac{p_{g}(x ; \pi)}{1-p_{g}(x ; \pi)}: \pi \in \Pi_{g}\right\}, \\
& \mathcal{H}_{2, g}=\left\{\left(x, c, y_{t}, y_{g-1}\right) \mapsto c \frac{p_{g}(x ; \pi)\left(y_{t}-y_{g-1}\right)}{1-p_{g}(x ; \pi)}: \pi \in \Pi_{g}\right\} \\
& \mathcal{H}_{3, g}=\left\{\left(x, c, y_{t}, y_{g-1}\right) \mapsto x \frac{c \dot{p}_{g}(x ; \pi)\left(y_{t}-y_{g-1}\right)}{\left(1-p_{g}(x ; \pi)\right)^{2}}: \pi \in \Pi_{g}\right\}, \\
& \mathcal{H}_{4, g}=\left\{(x, c) \mapsto x \frac{c \dot{p}_{g}(x ; \pi)}{\left(1-p_{g}(x ; \pi)\right)^{2}}: \pi \in \Pi_{g}\right\}, \\
& \mathcal{H}_{5, g}=\left\{\left(x, c, g_{g}\right) \mapsto x \frac{\left(g_{g}+c\right)\left(g_{g}-p_{g}(x ; \pi)\right) \dot{p}_{g}(x ; \pi)}{p_{g}(x ; \pi)\left(1-p_{g}(x ; \pi)\right)}: \pi \in \Pi_{g}\right\} .
\end{aligned}
$$

Lemma A.3. Under Assumptions 1 and 5 , for all $g=2, \ldots, \mathcal{T}, t=2, \ldots, \mathcal{T}$, the classes of 
functions $\mathcal{H}_{j, g}, j=\{1,2 \ldots, 5\}$, are Donsker.

Proof of Lemma A.3: This follows from Example 19.7 in van der Vaart (1998).

Lemma A.4. Under Assumptions 1 and 5, the null hypothesis

$$
H_{0}: \mathbb{E}\left[Y_{t}-Y_{t-1} \mid X, G_{g}=1\right]-\mathbb{E}\left[Y_{t}-Y_{t-1} \mid X, C=1\right]=0 \text { a.s.for all } 2 \leq t<g \leq \mathcal{T},
$$

can be equivalently written as

$$
H_{0}: \mathbb{E}\left[\left(\frac{G_{g}}{\mathbb{E}\left[G_{g}\right]}-\frac{\frac{p_{g}(X) C}{1-p_{g}(X)}}{\mathbb{E}\left[\frac{p_{g}(X) C}{1-p_{g}(X)}\right]}\right)\left(Y_{t}-Y_{t-1}\right) \mid X\right]=0 \text { a.s.for all } 2 \leq t<g \leq \mathcal{T} .
$$

Proof of Lemma A.4: First note that

$$
\begin{aligned}
\mathbb{E}\left[Y_{t}-Y_{t-1} \mid X, G_{g}=1\right] & =\mathbb{E}\left[G_{g}\left(Y_{t}-Y_{t-1}\right) \mid X, G_{g}=1\right] \\
& =\mathbb{E}\left[\frac{G_{g}}{\mathbb{E}\left[G_{g} \mid X\right]}\left(Y_{t}-Y_{t-1}\right) \mid X\right]
\end{aligned}
$$

Analogously,

$$
\mathbb{E}\left[Y_{t}-Y_{t-1} \mid X, C=1\right]=\mathbb{E}\left[\frac{C}{\mathbb{E}[C \mid X]}\left(Y_{t}-Y_{t-1}\right) \mid X\right]
$$

implying that

$$
\begin{aligned}
\mathbb{E}\left[Y_{t}-Y_{t-1} \mid X, G_{g}=1\right]-\mathbb{E}\left[Y_{t}-Y_{t-1} \mid X, C=1\right] & =0 \text { a.s.for all } 2 \leq t<g \leq \mathcal{T} . \\
& \Longleftrightarrow \\
\mathbb{E}\left[\left(\frac{G_{g}}{\mathbb{E}\left[G_{g} \mid X\right]}-\frac{C}{\mathbb{E}[C \mid X]}\right)\left(Y_{t}-Y_{t-1}\right) \mid X\right] & =0 \text { a.s.for all } 2 \leq t<g \leq \mathcal{T} .
\end{aligned}
$$

Given that under Assumptions 4 and $5, \mathbb{E}\left[G_{g}+C \mid X\right]>0$ a.s., we have that

$$
\mathbb{E}\left[\left(\frac{G_{g}}{\mathbb{E}\left[G_{g} \mid X\right]}-\frac{C}{\mathbb{E}[C \mid X]}\right)\left(Y_{t}-Y_{t-1}\right) \mid X\right]=0 \text { a.s.for all } 2 \leq t<g \leq \mathcal{T}
$$


if an only if

$$
\mathbb{E}\left[\mathbb{E}\left[G_{g}+C \mid X\right]\left(\frac{G_{g}}{\mathbb{E}\left[G_{g} \mid X\right]}-\frac{C}{\mathbb{E}[C \mid X]}\right)\left(Y_{t}-Y_{t-1}\right) \mid X\right]=0 \text { a.s.for all } 2 \leq t<g \leq \mathcal{T}
$$

By noticing that

$$
p_{g}(X)=\frac{\mathbb{E}\left[G_{g} \mid X\right]}{\mathbb{E}\left[G_{g}+C \mid X\right]}, 1-p_{g}(X)=\frac{\mathbb{E}[C \mid X]}{\mathbb{E}\left[G_{g}+C \mid X\right]},
$$

and that both of these are bounded away from zero under Assumption 5, we can rewrite (A.3) as

$$
\mathbb{E}\left[\left(\frac{G_{g}}{\mathbb{E}\left[G_{g}\right]}-\frac{\frac{p_{g}(X) C}{1-p_{g}(X)}}{\mathbb{E}\left[\frac{p_{g}(X) C}{1-p_{g}(X)}\right]}\right)\left(Y_{t}-Y_{t-1}\right) \mid X\right]=0 \text { a.s.for all } 2 \leq t<g \leq \mathcal{T},
$$

since

$$
\begin{aligned}
\mathbb{E}\left[\frac{p_{g}(X) C}{\left(1-p_{g}(X)\right)}\right] & =\mathbb{E}\left[\frac{\mathbb{E}\left[G_{g} \mid X, C+G_{g}=1\right] C}{\mathbb{E}\left[C \mid X, C+G_{g}=1\right]}\right] \\
& =\mathbb{E}\left[\frac{\mathbb{E}\left[G_{g} \mid X\right] C}{\mathbb{E}[C \mid X]}\right] \\
& =\mathbb{E}\left[\frac{\mathbb{E}\left[G_{g} \mid X\right] \mathbb{E}[C \mid X]}{\mathbb{E}[C \mid X]}\right] \\
& =\mathbb{E}\left[\mathbb{E}\left[G_{g} \mid X\right]\right] \\
& =\mathbb{E}\left[G_{g}\right] .
\end{aligned}
$$

This completes the proof.

Now, we are ready to proceed with the proofs of our main theorems.

Proof of Theorem 1: Given the result in Lemma A.1,

$$
\begin{aligned}
A T T(g, t) & =\mathbb{E}\left[A T T_{X}(g, t) \mid G_{g}=1\right] \\
& =\mathbb{E}\left[\mathbb{E}\left[Y_{t}-Y_{g-1} \mid X, G_{g}=1\right]-\mathbb{E}\left[Y_{t}-Y_{g-1} \mid X, C=1\right] \mid G_{g}=1\right] \\
& :=\mathbb{E}\left[A_{X} \mid G_{g}=1\right]-\mathbb{E}\left[B_{X} \mid G_{g}=1\right],
\end{aligned}
$$

and we consider each term separately. For the first term

$$
\mathbb{E}\left[A_{X} \mid G_{g}=1\right]=\mathbb{E}\left[Y_{t}-Y_{g-1} \mid G_{g}=1\right]
$$




$$
=\mathbb{E}\left[\frac{G_{g}}{\mathbb{E}\left[G_{g}\right]}\left(Y_{t}-Y_{g-1}\right)\right] .
$$

For the second term, by repetition of the law of iterated expectations, we have

$$
\begin{aligned}
& \mathbb{E}\left[B_{X} \mid G_{g}=1\right]=\mathbb{E}\left[\mathbb{E}\left[Y_{t}-Y_{g-1} \mid X, C=1\right] \mid G_{g}=1\right] \\
& =\mathbb{E}\left[G_{g} \mathbb{E}\left[C\left(Y_{t}-Y_{g-1}\right) \mid X, C=1\right] \mid G_{g}=1\right] \\
& =\mathbb{E}\left[G_{g} \mathbb{E}\left[\frac{C}{\left(1-p_{g}(X)\right)}\left(Y_{t}-Y_{g-1}\right) \mid X, G_{g}+C=1\right] \mid G_{g}=1\right] \\
& =\frac{\mathbb{E}\left[G_{g} \mathbb{E}\left[\frac{C}{\left(1-p_{g}(X)\right)}\left(Y_{t}-Y_{g-1}\right) \mid X, G_{g}+C=1\right] \mid G_{g}+C=1\right]}{\mathbb{E}\left[G_{g} \mid G_{g}+C=1\right]} \\
& =\frac{\mathbb{E}\left[\mathbb{E}\left[\frac{p_{g}(X) C}{\left(1-p_{g}(X)\right)}\left(Y_{t}-Y_{g-1}\right) \mid X, G_{g}+C=1\right] \mid G_{g}+C=1\right]}{\mathbb{E}\left[G_{g} \mid G_{g}+C=1\right]} \\
& =\mathbb{E}\left[G_{g}\right]^{-1} \mathbb{E}\left[\mathbb{E}\left[G_{g}+C \mid X\right] \mathbb{E}\left[\frac{p_{g}(X) C}{\left(1-p_{g}(X)\right)}\left(Y_{t}-Y_{g-1}\right) \mid X, G_{g}+C=1\right]\right] \\
& =\mathbb{E}\left[G_{g}\right]^{-1} \mathbb{E}\left[\mathbb{E}\left[\frac{p_{g}(X) C}{\left(1-p_{g}(X)\right)}\left(Y_{t}-Y_{g-1}\right) \mid X\right]\right] \\
& =\mathbb{E}\left[G_{g}\right]^{-1} \mathbb{E}\left[\mathbb{E}\left[\frac{p_{g}(X) C}{\left(1-p_{g}(X)\right)}\left(Y_{t}-Y_{g-1}\right)\right]\right] \\
& =\frac{\mathbb{E}\left[\frac{p_{g}(X) C}{\left(1-p_{g}(X)\right)}\left(Y_{t}-Y_{g-1}\right)\right]}{\mathbb{E}\left[\frac{p_{g}(X) C}{\left(1-p_{g}(X)\right)}\right]},
\end{aligned}
$$

where (A.6) follows from (A.4). The proof is completed by combining (A.5) and (A.6).

Proof of Theorem 2: Remember that

$$
\begin{aligned}
\widehat{A T T}(g, t) & =\mathbb{E}_{n}\left[\frac{G_{g}}{\mathbb{E}_{n}\left[G_{g}\right]}\left(Y_{t}-Y_{g-1}\right)\right]-\mathbb{E}_{n}\left[\frac{\frac{\hat{p}_{g}(X) C}{1-\hat{p}_{g}(X)}}{\mathbb{E}_{n}\left[\frac{\hat{p}_{g}(X) C}{1-\hat{p}_{g}(X)}\right]}\left(Y_{t}-Y_{g-1}\right)\right], \\
& :=\widehat{A T T}_{g}(g, t)-\widehat{A T T}_{C}(g, t),
\end{aligned}
$$


and

$$
\begin{aligned}
A T T(g, t) & =\mathbb{E}\left[\frac{G_{g}}{\mathbb{E}\left[G_{g}\right]}\left(Y_{t}-Y_{g-1}\right)\right]-\mathbb{E}\left[\frac{\frac{p_{g}(X) C}{1-p_{g}(X)}}{\mathbb{E}\left[\frac{p_{g}(X) C}{1-p_{g}(X)}\right]}\left(Y_{t}-Y_{g-1}\right)\right] \\
& :=\operatorname{ATT}_{g}(g, t)-\operatorname{ATT}_{C}(g, t) .
\end{aligned}
$$

In what follows we will separately show that, for $2 \leq g \leq t \leq \mathcal{T}$,

$$
\sqrt{n}\left(\widehat{A T T}_{g}(g, t)-A T T_{g}(g, t)\right)=\frac{1}{\sqrt{n}} \sum_{i=1}^{n} \psi_{g t}^{G}\left(\mathcal{W}_{i}\right)+o_{p}(1)
$$

and

$$
\sqrt{n}\left(\widehat{A T T}_{C}(g, t)-A T T_{C}(g, t)\right)=\frac{1}{\sqrt{n}} \sum_{i=1}^{n} \psi_{g t}^{C}\left(\mathcal{W}_{i}\right)+o_{p}(1)
$$

Then,

$$
\sqrt{n}(\widehat{A T T}(g, t)-A T T(g, t))=\frac{1}{\sqrt{n}} \sum_{i=1}^{n} \psi_{g t}\left(\mathcal{W}_{i}\right)+o_{p}(1)
$$

hold from (A.7) and (A.8), and the asymptotic normality result follows from the application of the central limit theorem.

Let $\beta_{g}=\mathbb{E}\left[G_{g}\right]$ and $\widehat{\beta}_{g}=\mathbb{E}_{n}\left[G_{g}\right]$, and note that

$$
\sqrt{n}\left(\widehat{\beta}_{g}-\beta_{g}\right)=\frac{1}{\sqrt{n}} \sum_{i=1}^{n}\left(G_{i g}-\mathbb{E}\left[G_{g}\right]\right) .
$$

Then, for all $2 \leq g \leq t \leq \mathcal{T}$, by the continuous mapping theorem,

$$
\begin{aligned}
\sqrt{n}\left(\widehat{A T T}_{g}(g, t)-A T T_{g}(g, t)\right) & =\frac{1}{\widehat{\beta}_{g}} \sqrt{n}\left(\mathbb{E}_{n}\left[G_{g}\left(Y_{t}-Y_{g-1}\right)\right]-\mathbb{E}\left[G_{g}\left(Y_{t}-Y_{g-1}\right)\right]\right) \\
& -\mathbb{E}\left[G_{g}\left(Y_{t}-Y_{g-1}\right)\right] \sqrt{n}\left(\frac{1}{\beta_{g}}-\frac{1}{\widehat{\beta}_{g}}\right) \\
& =\frac{1}{\beta_{g}} \frac{1}{\sqrt{n}} \sum_{i=1}^{n}\left(G_{i g}\left(Y_{i t}-Y_{i g-1}\right)-\mathbb{E}\left[G_{g}\left(Y_{t}-Y_{g-1}\right)\right]\right) \\
& -\frac{\mathbb{E}\left[G_{g}\left(Y_{t}-Y_{g-1}\right)\right]}{\beta_{g}^{2}} \sqrt{n}\left(\widehat{\beta}_{g}-\beta_{g}\right)+o_{p}(1) \\
& =\frac{1}{\sqrt{n}} \sum_{i=1}^{n}\left(\frac{G_{i g}\left(Y_{i t}-Y_{i g-1}\right)}{\beta_{g}}-\frac{G_{i g} \mathbb{E}\left[G_{g}\left(Y_{t}-Y_{g-1}\right)\right]}{\beta_{g}^{2}}\right)+o_{p}(1)
\end{aligned}
$$




$$
\begin{aligned}
& =\frac{1}{\sqrt{n}} \sum_{i=1}^{n} \frac{G_{i g}\left(\left(Y_{i t}-Y_{i g-1}\right)-A T T_{g}(g, t)\right)}{\beta_{g}}+o_{p}(1) \\
& :=\frac{1}{\sqrt{n}} \sum_{i=1}^{n} \psi_{g t}^{G}\left(\mathcal{W}_{i}\right)+o_{p}(1),
\end{aligned}
$$

concluding the proof of (A.7).

Next we focus on (A.8). For an arbitrary function $g$, let

$$
w(g)=\frac{g(X) C}{1-g(X)},
$$

and note that

$$
\begin{aligned}
\sqrt{n}\left(\widehat{A T T}_{C}(g, t)-A T T_{C}(g, t)\right) & =\frac{1}{\mathbb{E}_{n}\left[w\left(\hat{p}_{g}\right)\right]} \sqrt{n}\left(\mathbb{E}_{n}\left[w\left(\hat{p}_{g}\right)\left(Y_{t}-Y_{g-1}\right)\right]-\mathbb{E}\left[w\left(p_{g}\right)\left(Y_{t}-Y_{g-1}\right)\right]\right) \\
& -\frac{\mathbb{E}\left[w\left(p_{g}\right)\left(Y_{t}-Y_{g-1}\right)\right]}{\mathbb{E}_{n}\left[w\left(\hat{p}_{g}\right)\right] \mathbb{E}\left[w\left(p_{g}\right)\right]} \sqrt{n}\left(\mathbb{E}_{n}\left[w\left(\hat{p}_{g}\right)\right]-\mathbb{E}\left[w\left(p_{g}\right)\right]\right) \\
& :=\frac{1}{\mathbb{E}_{n}\left[w\left(\hat{p}_{g}\right)\right]} \cdot \sqrt{n} A_{n}\left(\hat{p}_{g}\right)-\frac{A T T_{C}(g, t)}{\mathbb{E}_{n}\left[w\left(\hat{p}_{g}\right)\right]} \cdot \sqrt{n} B_{n}\left(\hat{p}_{g}\right) .
\end{aligned}
$$

From Assumption 5, Lemmas A.2 and A.3, and the continuous mapping theorem,

$$
\begin{aligned}
\frac{1}{\mathbb{E}_{n}\left[w\left(\hat{p}_{g}\right)\right]} & =\frac{1}{\mathbb{E}\left[w\left(p_{g}\right)\right]}+o_{p}(1), \\
\frac{A T T_{C}(g, t)}{\mathbb{E}_{n}\left[w\left(\hat{p}_{g}\right)\right]} & =\frac{A T T_{C}(g, t)}{\mathbb{E}\left[w\left(p_{g}\right)\right]}+o_{p}(1) .
\end{aligned}
$$

Thus,

$$
\begin{aligned}
\sqrt{n}\left(\widehat{A T T}_{C}(g, t)-A T T_{C}(g, t)\right)=\frac{1}{\mathbb{E}\left[w\left(p_{g}\right)\right]} \cdot \sqrt{n} A_{n}\left(\hat{p}_{g}\right) & \\
& -\frac{A T T_{C}(g, t)}{\mathbb{E}\left[w\left(p_{g}\right)\right]} \cdot \sqrt{n} B_{n}\left(\hat{p}_{g}\right)+o_{p}(1)
\end{aligned}
$$

Applying a classical mean value theorem argument,

$$
\begin{aligned}
A_{n}\left(\hat{p}_{g}\right) & =\mathbb{E}_{n}\left[w\left(p_{g}\right)\left(Y_{t}-Y_{g-1}\right)\right]-\mathbb{E}\left[w\left(p_{g}\right)\left(Y_{t}-Y_{g-1}\right)\right] \\
& +\mathbb{E}_{n}\left[X\left(\frac{C}{1-p_{g}\left(X ; \bar{\pi}_{g}\right)}\right)^{2} \dot{p}_{g}\left(X ; \bar{\pi}_{g}\right)\left(Y_{i t}-Y_{i g-1}\right)\right]^{\prime}\left(\hat{\pi}_{g}-\pi_{g}^{0}\right),
\end{aligned}
$$

where $\bar{\pi}$ is an intermediate point that satisfies $\left|\bar{\pi}_{g}-\pi_{g}^{0}\right| \leq\left|\hat{\pi}_{g}-\pi_{g}^{0}\right|$ a.s. Thus, by Assumption 5, 
Lemmas A.2 and A.3, and the Classical Glivenko-Cantelli's theorem,

$$
\begin{aligned}
A_{n}\left(\hat{p}_{g}\right) & =\mathbb{E}_{n}\left[w\left(p_{g}\right)\left(Y_{t}-Y_{g-1}\right)-\mathbb{E}\left[w\left(p_{g}\right)\left(Y_{t}-Y_{g-1}\right)\right]\right] \\
& +\mathbb{E}\left[X\left(\frac{C}{1-p_{g}(X)}\right)^{2} \dot{p}_{g}(X)\left(Y_{i t}-Y_{i g-1}\right)\right]^{\prime}\left(\hat{\pi}_{g}-\pi_{g}^{0}\right)+o_{p}\left(n^{-1 / 2}\right) .
\end{aligned}
$$

Analogously,

$$
\begin{aligned}
B_{n}\left(\hat{p}_{g}\right) & =\mathbb{E}_{n}\left[w\left(p_{g}\right)-\mathbb{E}\left[w\left(p_{g}\right)\right]\right] \\
& +\mathbb{E}\left[X\left(\frac{C}{1-p_{g}(X)}\right)^{2} \dot{p}_{g}(X)\right]^{\prime}\left(\hat{\pi}_{g}-\pi_{g}^{0}\right)+o_{p}\left(n^{-1 / 2}\right) .
\end{aligned}
$$

Then, (A.9), (A.10), (A.12) and Lemma A.2 yield (A.8), concluding the proof. $\square$

Proof of Theorem 3: Note that, by the conditional multiplier central limit theorem, see Lemma 2.9.5 in van der Vaart and Wellner (1996), as $n \rightarrow \infty$,

$$
\frac{1}{\sqrt{n}} \sum_{i=1}^{n} V_{i} \cdot \Psi_{g \leq t}\left(\mathcal{W}_{i}\right) \stackrel{d}{\rightarrow} N(0, \Sigma)
$$

where $\Sigma=\mathbb{E}\left[\Psi_{g \leq t}(\mathcal{W}) \Psi_{g \leq t}(\mathcal{W})^{\prime}\right]$. Thus, to conclude the proof that

$$
\sqrt{n}\left(\widehat{A T T}_{g \leq t}^{*}-\widehat{A T T}_{g \leq t}\right) \underset{*}{\stackrel{d}{\rightarrow}} N(0, \Sigma)
$$

it suffices to show that, for all $2 \leq g \leq t \leq \mathcal{T}$,

$$
\frac{1}{\sqrt{n}} \sum_{i=1}^{n} V_{i} \cdot\left[\widehat{\psi}_{g t}\left(\mathcal{W}_{i}\right)-\psi_{g t}\left(\mathcal{W}_{i}\right)\right]=o_{p^{*}}(1)
$$

Towards this, note that

$$
\begin{aligned}
\frac{1}{\sqrt{n}} \sum_{i=1}^{n} V_{i} \cdot\left[\widehat{\psi}_{g t}\left(\mathcal{W}_{i}\right)-\psi_{g t}\left(\mathcal{W}_{i}\right)\right]= & \frac{1}{\sqrt{n}} \sum_{i=1}^{n} V_{i} \cdot\left[\widehat{\psi}_{g t}^{G}\left(\mathcal{W}_{i}\right)-\psi_{g t}^{G}\left(\mathcal{W}_{i}\right)\right] \\
& -\frac{1}{\sqrt{n}} \sum_{i=1}^{n} V_{i} \cdot\left[\widehat{\psi}_{g t}^{C}\left(\mathcal{W}_{i}\right)-\psi_{g t}^{C}\left(\mathcal{W}_{i}\right)\right],
\end{aligned}
$$

where

$$
\widehat{\psi}_{g t}^{G}(\mathcal{W})=\frac{G_{g}}{\mathbb{E}_{n}\left[G_{g}\right]}\left[\left(Y_{t}-Y_{g-1}\right)-\widehat{A T T}_{g}(g, t)\right],
$$


and

$$
\widehat{\psi}_{g t}^{C}(\mathcal{W})=\frac{w\left(\hat{p}_{g}\right)}{\mathbb{E}_{n}\left[w\left(\hat{p}_{g}\right)\right]}\left[\left(Y_{i t}-Y_{i g-1}\right)-\widehat{A T T}_{C}(g, t)\right]+\widehat{M}_{g t}^{\prime} \widehat{\xi}_{g}^{\pi}(\mathcal{W})
$$

with

$$
\begin{aligned}
w\left(\hat{p}_{g}\right) & =\frac{\hat{p}_{g}(X) C}{1-\hat{p}_{g}(X)} \\
\widehat{M}_{g t} & =\frac{\mathbb{E}_{n}\left[X\left(\frac{C}{1-\hat{p}_{g}(X)}\right)^{2} \widehat{\dot{p}}_{g}(X)\left[\left(Y_{i t}-Y_{i g-1}\right)-\widehat{A T T}_{g}(g, t)\right]\right]}{\mathbb{E}_{n}\left[w\left(\hat{p}_{g}\right)\right]} \\
\widehat{\xi}_{g}^{\pi}(\mathcal{W}) & =\mathbb{E}_{n}\left[\frac{\left(G_{g}+C\right) \widehat{\dot{p}}_{g}(X)^{2}}{\hat{p}_{g}(X)\left(1-\hat{p}_{g}(X)\right)} X X^{\prime}\right]^{-1} X \frac{\left(G_{g}+C\right)\left(G_{g}-\hat{p}_{g}(X)\right) \widehat{\dot{p}}_{g}(X)}{\hat{p}_{g}(X)\left(1-\hat{p}_{g}(X)\right)} .
\end{aligned}
$$

We will show that each term in (A.14) is $o_{p^{*}}(1)$. For the first term in (A.14), we have

$$
\begin{aligned}
& \frac{1}{\sqrt{n}} \sum_{i=1}^{n} V_{i} \cdot\left[\widehat{\psi}_{g t}^{G}\left(\mathcal{W}_{i}\right)-\psi_{g t}^{G}\left(\mathcal{W}_{i}\right)\right] \\
& =\left[\frac{1}{\mathbb{E}_{n}\left[G_{g}\right]}-\frac{1}{\mathbb{E}\left[G_{g}\right]}\right] \frac{1}{\sqrt{n}} \sum_{i=1}^{n} V_{i} \cdot G_{i g}\left(Y_{i t}-Y_{i g-1}\right) \\
& -\left[\widehat{A T T}_{g}(g, t)-A T T_{g}(g, t)\right] \frac{1}{\sqrt{n}} \sum_{i=1}^{n} V_{i} \cdot G_{i g}, \\
& =o_{p^{*}}(1)
\end{aligned}
$$

where the last equality follows from the results in Theorem 1, together with the law of large numbers, continuous mapping theorem, and Lemma 2.9.5 in van der Vaart and Wellner (1996).

For the second term in (A.14), we have

$$
\begin{aligned}
& \frac{1}{\sqrt{n}} \sum_{i=1}^{n} V_{i} \cdot\left[\widehat{\psi}_{g t}^{C}\left(\mathcal{W}_{i}\right)-\psi_{g t}^{C}\left(\mathcal{W}_{i}\right)\right] \\
& =\frac{1}{\mathbb{E}_{n}\left[w\left(\hat{p}_{g}\right)\right]} \frac{1}{\sqrt{n}} \sum_{i=1}^{n} V_{i} \cdot\left(w_{i}\left(\hat{p}_{g}\right)-w_{i}\left(p_{g}\right)\right)\left(Y_{i t}-Y_{i g-1}\right) \\
& +\left(\frac{1}{\mathbb{E}_{n}\left[w\left(\hat{p}_{g}\right)\right]}-\frac{1}{\mathbb{E}\left[w\left(p_{g}\right)\right]}\right) \frac{1}{\sqrt{n}} \sum_{i=1}^{n} V_{i} \cdot w_{i}\left(p_{g}\right)\left(Y_{i t}-Y_{i g-1}\right) \\
& +\left(\widehat{M}_{g t}-M_{g t}\right) \frac{1}{\sqrt{n}} \sum_{i=1}^{n} V_{i} \cdot \xi_{g}^{\pi}\left(\mathcal{W}_{i}\right) . \\
& +\widehat{M}_{g t} \frac{1}{\sqrt{n}} \sum_{i=1}^{n} V_{i} \cdot\left(\widehat{\xi}_{g}^{\pi}\left(\mathcal{W}_{i}\right)-\xi_{g}^{\pi}\left(\mathcal{W}_{i}\right)\right)
\end{aligned}
$$




$$
:=A_{1 n}+A_{2 n}+A_{3 n}+A_{4 n}
$$

From Lemma A.3, we have that $\mathcal{H}_{1, g}, \mathcal{H}_{2, g}, \mathcal{H}_{3, g}$ and $\mathcal{H}_{5, g}$ are Donsker, and by Assumption 5 , $\mathbb{E}\left[w\left(p_{g}\right)\right]$ it is bounded away from zero. Thus, by a stochastic equicontinuity argument, GlivenkoCantelli's theorem, continuous mapping theorem, and Theorem 2.9.6 in van der Vaart and Wellner (1996),

$$
A_{1 n}=o_{p^{*}}(1), A_{2 n}=o_{p^{*}}(1), A_{3 n}=o_{p^{*}}(1), \text { and } A_{4 n}=o_{p^{*}}(1),
$$

implying that

$$
\frac{1}{\sqrt{n}} \sum_{i=1}^{n} V_{i} \cdot\left[\widehat{\psi}_{g t}^{C}\left(\mathcal{W}_{i}\right)-\psi_{g t}^{C}\left(\mathcal{W}_{i}\right)\right]=o_{p^{*}}(1) .
$$

From (A.13)-(A.16), it follows that

$$
\sqrt{n}\left(\widehat{A T T}_{g \leq t}^{*}-\widehat{A T T}_{g \leq t}\right) \underset{*}{\rightarrow} N(0, \Sigma) .
$$

Finally, by the continuous mapping theorem, see e.g. Theorem 10.8 in Kosorok (2008), for any continuous functional $\Gamma(\cdot)$

$$
\Gamma\left(\sqrt{n}\left(\widehat{A T T}_{g \leq t}^{*}-\widehat{A T T}_{g \leq t}\right)\right) \underset{*}{\stackrel{d}{\rightarrow}} \Gamma(N(0, V))
$$

concluding our proof.

Proof of Theorem 4: In order to prove the first part of Theorem 4, we first show that, under $H_{0}$, for all $2 \leq t<g \leq \mathcal{T}$,

$$
\widehat{J}\left(u, g, t, \hat{p}_{g}\right)=\mathbb{E}_{n}\left[\psi_{\text {ugt }}^{\text {test }}\left(\mathcal{W}_{i}\right)\right]+o_{p}\left(n^{-1 / 2}\right),
$$

Towards this end, we write

$$
\begin{aligned}
\widehat{J}\left(u, g, t, \hat{p}_{g}\right) & =\mathbb{E}_{n}\left[\frac{G_{g}}{\mathbb{E}_{n}\left[G_{g}\right]} 1(X \leq u)\left(Y_{t}-Y_{t-1}\right)\right] \\
& -\mathbb{E}_{n}\left[\frac{\frac{\hat{p}_{g}(X) C}{1-\hat{p}_{g}(X)}}{\mathbb{E}_{n}\left[\frac{\hat{p}_{g}(X) C}{1-\hat{p}_{g}(X)}\right]} 1(X \leq u)\left(Y_{t}-Y_{t-1}\right)\right] \\
& :=\widehat{J}_{G}\left(u, g, t, \hat{p}_{g}\right)-\widehat{J}_{C}\left(u, g, t, \hat{p}_{g}\right),
\end{aligned}
$$


and analyze each term separately.

As in the proof of Theorem 1 , let $\beta_{g}=\mathbb{E}\left[G_{g}\right]$ and $\widehat{\beta}_{g}=\mathbb{E}_{n}\left[G_{g}\right]$. Applying a classical mean value theorem argument, uniformly in $u \in \mathcal{X}$,

$$
\begin{aligned}
\widehat{J}_{G}\left(u, g, t, \hat{p}_{g}\right) & =\mathbb{E}_{n}\left[\frac{G_{g}}{\beta_{g}} 1(X \leq u)\left(Y_{t}-Y_{t-1}\right)\right] \\
& -\frac{\mathbb{E}_{n}\left[G_{g} 1(X \leq u)\left(Y_{t}-Y_{t-1}\right)\right]}{\bar{\beta}_{g}^{2}} \cdot \mathbb{E}_{n}\left[G_{g}-\mathbb{E}\left[G_{g}\right]\right]
\end{aligned}
$$

where $\bar{\beta}_{g}$ is an intermediate point that satisfies $\left|\bar{\beta}_{g}-\beta_{g}\right| \leq\left|\widehat{\beta}_{g}-\beta_{g}\right|$ a.s.. Define the class of functions

$$
\mathcal{H}_{6, g}=\left\{\left(x, g_{g}, y_{t}, y_{t-1}\right) \mapsto g_{g}\left(y_{t}-y_{t-1}\right) 1\{x \leq u\}: u \in \mathcal{X}\right\}
$$

By Example 19.11 in van der Vaart (1998), $\mathcal{H}_{6, g}$ is Donsker under Assumption 5. Furthermore,

$$
\mathbb{E}_{n}\left[G_{g}-\mathbb{E}\left[G_{g}\right]\right]=O_{p}\left(n^{-1 / 2}\right)
$$

Thus, by the Glivenko-Cantelli's theorem and the continuous mapping theorem, uniformly in $u \in \mathcal{X}$

$$
\begin{aligned}
\widehat{J}_{G}\left(u, g, t, \hat{p}_{g}\right) & =\mathbb{E}_{n}\left[\frac{G_{g}}{\mathbb{E}\left[G_{g}\right]} 1(X \leq u)\left(Y_{t}-Y_{t-1}\right)\right] \\
& -\frac{J_{G}\left(u, g, t, p_{g}\right)}{\mathbb{E}\left[G_{g}\right]} \cdot \mathbb{E}_{n}\left[G_{g}-\mathbb{E}\left[G_{g}\right]\right]+o_{p}\left(n^{-1 / 2}\right) \\
& =\mathbb{E}_{n}\left[w_{g}^{G}\left(\left(Y_{t}-Y_{t-1}\right) 1(X \leq u)-\mathbb{E}\left[w_{g}^{G} 1(X \leq u)\left(Y_{t}-Y_{t-1}\right)\right]\right)\right]+J_{G}\left(u, g, t, p_{g}\right) \\
& +o_{p}\left(n^{-1 / 2}\right)
\end{aligned}
$$

where

$$
J_{G}\left(u, g, t, p_{g}\right)=\mathbb{E}\left[\frac{G_{g}}{\mathbb{E}\left[G_{g}\right]} 1(X \leq u)\left(Y_{t}-Y_{t-1}\right)\right] .
$$

We analyze $\widehat{J}_{C}\left(u, g, t, \hat{p}_{g}\right)$ next. Applying a classical mean value theorem argument, uniformly in $u \in \mathcal{X}$,

$$
\widehat{J}_{C}\left(u, g, t, \hat{p}_{g}\right)=\widehat{J}_{C}\left(u, g, t, p_{g}\right)
$$




$$
\begin{aligned}
& +\frac{\mathbb{E}_{n}\left[X \frac{C \dot{p}_{g}\left(X ; \bar{\pi}_{g}\right)}{\left(1-p_{g}\left(X ; \bar{\pi}_{g}\right)\right)^{2}} 1(X \leq u)\left(Y_{t}-Y_{t-1}\right)\right]^{\prime}}{\mathbb{E}_{n}\left[\frac{p_{g}\left(X ; \bar{\pi}_{g}\right) C}{1-p_{g}\left(X ; \bar{\pi}_{g}\right)}\right]}\left(\hat{\pi}_{g}-\pi_{g}^{0}\right) \\
& -\frac{\mathbb{E}_{n}\left[X \frac{C \dot{p}_{g}\left(X ; \bar{\pi}_{g}\right)}{\left(1-p_{g}\left(X ; \bar{\pi}_{g}\right)\right)^{2}}\right]^{\prime}}{\mathbb{E}_{n}\left[\frac{p_{g}\left(X ; \bar{\pi}_{g}\right) C}{1-p_{g}\left(X ; \bar{\pi}_{g}\right)}\right]} \frac{\mathbb{E}_{n}\left[\frac{p_{g}\left(X ; \bar{\pi}_{g}\right) C}{1-p_{g}\left(X ; \bar{\pi}_{g}\right)} 1(X \leq u)\left(Y_{t}-Y_{t-1}\right)\right]}{\mathbb{E}_{n}\left[\frac{p_{g}\left(X ; \bar{\pi}_{g}\right) C}{1-p_{g}\left(X ; \bar{\pi}_{g}\right)}\right]}\left(\hat{\pi}_{g}-\pi_{g}^{0}\right)
\end{aligned}
$$

where $\bar{\pi}$ is an intermediate point that satisfies $\left|\bar{\pi}_{g}-\pi_{g}^{0}\right| \leq\left|\hat{\pi}_{g}-\pi_{g}^{0}\right| a . s$, and

$$
\widehat{J}_{C}\left(u, g, t, p_{g}\right)=\frac{\mathbb{E}_{n}\left[\frac{p_{g}(X) C}{1-p_{g}(X)} 1(X \leq u)\left(Y_{t}-Y_{t-1}\right)\right]}{\mathbb{E}_{n}\left[\frac{p_{g}(X) C}{1-p_{g}(X)}\right]} .
$$

Define the classes of functions

$$
\begin{aligned}
\mathcal{H}_{7, g} & =\left\{\left(x, c, y_{t}, y_{t-1}\right) \mapsto \frac{p_{g}(x ; \pi)}{1-p_{g}(x ; \pi)} c\left(y_{t}-y_{t-1}\right) 1\{x \leq u\}: \pi \in \Pi_{g}, u \in \mathcal{X}\right\}, \\
\mathcal{H}_{8, g} & =\left\{\left(x, c, y_{t}, y_{t-1}\right) \mapsto x \frac{\dot{p}_{g}(x ; \pi) c\left(y_{t}-y_{t-1}\right) 1\{x \leq u\}}{\left(1-p_{g}(x ; \pi)\right)^{2}}: \pi \in \Pi_{g}, u \in \mathcal{X}\right\}, \\
\mathcal{H}_{9, g} & =\left\{(x, c) \mapsto \frac{c p_{g}(x ; \pi)}{1-p_{g}(x ; \pi)}: \pi \in \Pi_{g},\right\}, \\
\mathcal{H}_{10, g} & =\left\{(x, c) \mapsto x \frac{\dot{p}_{g}(x ; \pi) c}{\left(1-p_{g}(x ; \pi)\right)^{2}}: \pi \in \Pi_{g}\right\} .
\end{aligned}
$$

By Examples 19.7, 19.11, and 19.20 in van der Vaart (1998), all these classes of functions are Donsker under Assumption 5. Thus, by the Glivenko-Cantelli's theorem, continuous mapping theorem, and Lemma A.2, uniformly in $u \in \mathcal{X}$,

$$
\widehat{J}_{C}\left(u, g, t, \hat{p}_{g}\right)=\widehat{J}_{C}\left(u, g, t, p_{g}\right)+M_{u g t}^{\text {test }}\left(\hat{\pi}_{g}-\pi_{g}^{0}\right)+o_{p}\left(n^{-1 / 2}\right),
$$

for every $g, t$.

Denote

$$
\hat{\beta}_{g}^{C}=\mathbb{E}_{n}\left[\frac{p_{g}(X) C}{1-p_{g}(X)}\right], \beta_{g}^{C}=\mathbb{E}\left[\frac{p_{g}(X) C}{1-p_{g}(X)}\right] .
$$


Applying a classical mean value theorem argument, we have

$$
\begin{aligned}
\widehat{J}_{C}\left(u, g, t, p_{g}\right) & =\frac{\mathbb{E}_{n}\left[\frac{p_{g}(X) C}{1-p_{g}(X)} 1(X \leq u)\left(Y_{t}-Y_{t-1}\right)\right]}{\mathbb{E}\left[\frac{p_{g}(X) C}{1-p_{g}(X)}\right]} \\
& -\frac{\mathbb{E}_{n}\left[\frac{p_{g}(X) C}{1-p_{g}(X)} 1(X \leq u)\left(Y_{t}-Y_{t-1}\right)\right]}{\left(\bar{\beta}_{g}^{C}\right)^{2}} \cdot \mathbb{E}_{n}\left[\frac{p_{g}(X) C}{1-p_{g}(X)}-\mathbb{E}\left[\frac{p_{g}(X) C}{1-p_{g}(X)}\right]\right]
\end{aligned}
$$

where $\bar{\beta}_{g}^{C}$ is an intermediate point that satisfies $\left|\bar{\beta}_{g}^{C}-\beta_{g}^{C}\right| \leq\left|\widehat{\beta}_{g}^{C}-\beta_{g}^{C}\right|$ a.s.. Since $\mathcal{H}_{7, g}$ is a Donsker Class of functions and

$$
\mathbb{E}_{n}\left[\frac{p_{g}(X) C}{1-p_{g}(X)}-\mathbb{E}\left[\frac{p_{g}(X) C}{1-p_{g}(X)}\right]\right]=O_{p}\left(n^{-1 / 2}\right)
$$

we have that, by the Glivenko-Cantelli's theorem and the continuous mapping theorem, uniformly in $u \in \mathcal{X}$,

$$
\begin{aligned}
\widehat{J}_{C}\left(u, g, t, p_{g}\right) & =\mathbb{E}_{n}\left[w_{g}^{C}\left(Y_{t}-Y_{t-1}\right) 1(X \leq u)\right] \\
& -\frac{\mathbb{E}\left[w_{g}^{C}\left(Y_{t}-Y_{t-1}\right) 1(X \leq u)\right]}{\mathbb{E}\left[\frac{p_{g}(X) C}{1-p_{g}(X)}\right]} \cdot \mathbb{E}_{n}\left[\frac{p_{g}(X) C}{1-p_{g}(X)}-\mathbb{E}\left[\frac{p_{g}(X) C}{1-p_{g}(X)}\right]\right]+o_{p}\left(n^{-1 / 2}\right) \\
& =\mathbb{E}_{n}\left[w_{g}^{C}\left(\left(Y_{t}-Y_{t-1}\right) 1(X \leq u)-\mathbb{E}\left[w_{g}^{C} 1(X \leq u)\left(Y_{t}-Y_{t-1}\right)\right]\right)\right]+J_{C}\left(u, g, t, p_{g}\right) \\
& +o_{p}\left(n^{-1 / 2}\right)
\end{aligned}
$$

Hence, from (A.17), (A.18), (A.19), and the asymptotic linear representation of $\left(\hat{\pi}_{g}-\pi_{g}^{0}\right)$ in Lemma A.2, for every $g, t$,

$$
\widehat{J}\left(u, g, t, \hat{p}_{g}\right)=\mathbb{E}_{n}\left[\psi_{\text {ugt }}^{\text {test }}(\mathcal{W})\right]+\left(J_{G}\left(u, g, t, p_{g}\right)-J_{C}\left(u, g, t, p_{g}\right)\right)+o_{p}\left(n^{-1 / 2}\right)
$$

By noticing that under $H_{0}, J_{G}\left(u, g, t, p_{g}\right)=J_{C}\left(u, g, t, p_{g}\right)$ for all $u \in \mathcal{X},(g, t)$ such that $2 \leq t<g \leq \mathcal{T}$, we have that, under $H_{0}$, uniformly in $u \in \mathcal{X}$, for all $2 \leq t<g \leq \mathcal{T}$

$$
\widehat{J}\left(u, g, t, \hat{p}_{g}\right)=\mathbb{E}_{n}\left[\psi_{\text {ugt }}^{\text {test }}\left(\mathcal{W}_{i}\right)\right]+o_{p}\left(n^{-1 / 2}\right)
$$


In order to show that $\sqrt{n} \widehat{J}_{g>t}(u) \Rightarrow \mathbb{G}(u)$ in $l^{\infty}(\mathcal{X})$, it suffices to show that the class of functions

$$
\mathcal{H}_{10}=\left\{\left(x, g_{g}, c, y_{t}, y_{t-1}\right) \mapsto \psi_{\text {ugt }}^{\text {test }}: u \in \mathcal{X}, 2 \leq t<g \leq \mathcal{T}\right\}
$$

is Donsker. This follows straightforwardly from the the previously discussed Donsker results and Example 19.20 in van der Vaart (1998). Finally,

$$
C v M_{n} \stackrel{d}{\rightarrow} \int_{\mathcal{X}}|\mathbb{G}(u)|_{M}^{2} F_{X}(d u)
$$

follows from the continuous mapping theorem,

$$
\sup _{u \in \mathcal{X}}\left|F_{n, X}(u)-F_{X}(u)\right|=o_{a . s .}(1)
$$

and the Helly-Bray Theorem.

Next, we study the behavior of $C v M_{n}$ under $H_{1}$. First, note that under $H_{1}$, for some $u \in \mathcal{X}$, and some $(g, t), 2 \leq t<g \leq \mathcal{T}$,

$$
J\left(u, g, t, p_{g}\right) \neq 0 \text {. }
$$

Thus, from (A.20), under $H_{1}$, uniformly in $u \in \mathcal{X}$,

$$
\sqrt{n} \widehat{J}_{g>t}(u)=O_{p}\left(n^{1 / 2}\right)
$$

implying that $C v M_{n}$ diverges to infinity under $H_{1}$. Because $c_{\alpha}^{C v M}=O(1)$ a.s., as $n \rightarrow \infty$,

$$
P\left(C v M_{n}>c_{\alpha}^{C v M}\right) \rightarrow 1
$$

concluding the proof of Theorem 4 .

Proof of Theorem 5: In the proof of Theorem 4, we have shown that

$$
\mathcal{H}_{11}=\left\{\left(x, g_{g}, c, y_{t}, y_{t-1}\right) \mapsto \psi_{\text {ugt }}^{\text {test }}: u \in \mathcal{X}, 2 \leq t<g \leq \mathcal{T}\right\}
$$

is a Donsker class of functions. Then, by the conditional multiplier functional central limit theorem, see Theorem 2.9.6, in van der Vaart and Wellner (1996), as $n \rightarrow \infty$,

$$
\frac{1}{\sqrt{n}} \sum_{i=1}^{n} V_{i} \cdot \Psi_{g>t}^{\text {test }}\left(\mathcal{W}_{i}\right) \underset{*}{\Rightarrow} \mathbb{G}(u) \text { in } l^{\infty}(\mathcal{X})
$$


where $\mathbb{G}(u)$ in $l^{\infty}(\mathcal{X})$ is the same Gaussian process of Theorem 4 and $\underset{*}{\Rightarrow}$ indicates weak convergence in probability under the bootstrap law. Thus, to conclude the proof it suffices to show that, for all $2 \leq t<g \leq \mathcal{T}$, uniformly in $u \in \mathcal{X}$,

$$
\frac{1}{\sqrt{n}} \sum_{i=1}^{n} V_{i} \cdot\left[\widehat{\psi}_{\text {ugt }}^{\text {test }}\left(\mathcal{W}_{i}\right)-\psi_{\text {ugt }}^{\text {test }}\left(\mathcal{W}_{i}\right)\right]=o_{p^{*}}(1)
$$

The proof of (A.21) follows exactly the same steps as the proof of Theorem (3), and is therefore omitted.

\section{References}

Abadie, A. (2005), "Semiparametric difference-in-difference estimators," Review of Economic Studies, $72,1-19$.

Amemiya, T. (1985), Advanced Econometrics, Cambridge: Harvard University Press.

Angrist, J. D., and Pischke, J.-S. (2008), Mostly Harmless Econometrics: An Empiricist's Companion, : Princeton University Press.

Athey, S., and Imbens, G. W. (2006), "Identification and inference in nonlinear difference in differences models," Econometrica, 74(2), 431-497.

Autor, D. H., Kerr, W. R., and Kugler, A. D. (2007), "Does Employment Protection Reduce Productivity? Evidence From US States," The Economic Journal, 117(521), F189-F217.

Belloni, A., Chernozhukov, V., Fernandez-Val, I., and Hansen, C. (2017), "Program Evaluation and Causal Inference With High-Dimensional Data," Econometrica, 85(1), 233-298.

Belman, D., and Wolfso, P. J. (2014), What does the minimum wage do?, Kalamazoo, MI: W.E. Upjohn Institute for Employment Research.

Bertrand, M., Duflo, E., and Mullainathan, S. (2004), "How Much Should We Trust DifferencesIn-Differences Estimates?," The Quarterly Journal of Economics, 119(1), 249-275.

Bierens, H. J. (1982), "Consistent model specification tests," Journal of Econometrics, 20(1982), 105-134.

Bierens, H. J., and Ploberger, W. (1997), "Asymptotic theory of integrated conditional moment tests," Econometrica, 65(5), 1129-1151.

Blundell, R., Dias, M. C., Meghir, C., and van Reenen, J. (2004), "Evaluating the Employment Impact of a Mandatory Job Search Program," Journal of the European Economic Association, 2(4), 569-606.

Bonhomme, S., and Sauder, U. (2011), "Recovering distributions in difference-in-differences models: a comparison of selective and comprehensive schooling," , 93(May), 479-494.

Borusyak, K., and Jaravel, X. (2017), "Revisiting Event Study Designs," Mimeo, pp. 1-33. 
Botosaru, I., and Gutierrez, F. H. (2017), "Difference-in-differences when the treatment status is observed in only one period," Journal of Applied Econometrics, (March 2017), 73-90.

Busso, M., Dinardo, J., and McCrary, J. (2014), "New Evidence on the Finite Sample Properties of Propensity Score Reweighting and Matching Estimators," The Review of Economics and Statistics, 96(5), 885-895.

Callaway, B., Li, T., and Oka, T. (2018), "Quantile Treatment Effects in Difference in Differences Models Under Dependence Restrictions and with Only Two Time Periods," Journal of Econometrics, Forthcoming.

Card, D., and Krueger, A. B. (1994), "Minimum Wages and Employment: A Case Study of the Fast-Food Industry in New Jersey and Pennsylvania," American Economic Review, 84(4), 772793.

Cheng, G., Yu, Z., and Huang, J. Z. (2013), "The cluster bootstrap consistency in generalized estimating equations," Journal of Multivariate Analysis, 115, 33-47.

Chernozhukov, V., Fernandez-Val, I., Hahn, J., and Newey, W. (2013), "Average and Quantile Effects in Nonseparable Panel Models," Econometrica, 81(2), 535-580.

Chernozhukov, V., Fernandez-Val, I., and Luo, Y. (2017), "The sorted effects method: discovering heterogeneous effects beyond their averages," Arxiv preprint arXiv:1512.05635, .

de Chaisemartin, C., and D'Haultfoeuille, X. (2016), "Double fixed effects estimators with heterogeneous treatment effects," Working Paper, (Did), 1-23.

de Chaisemartin, C., and D'Haultfœuille, X. (2017), "Fuzzy Differences-in-Differences," The Review of Economic Studies, (February), 1-30.

Donald, S. G., and Hsu, Y.-C. (2014), "Estimation and inference for distribution functions and quantile functions in treatment effect models," Journal of Econometrics, 178(3), 383-397.

Doucouliagos, H., and Stanley, T. D. (2009), "Publication selection bias in minimum-wage research? A meta-regression analysis," British Journal of Industrial Relations, 47(2), 406-428.

Dube, A., Lester, T. W., and Reich, M. (2010), "Minimum Wage Effects Across State Borders: Estimates Using Contiguous Counties," Review of Economics and Statistics, 92(4), 945-964.

Dube, A., Lester, T. W., and Reich, M. (2016), "Minimum wage shocks, employment flows, and labor market frictions," Journal of Labor Economics, 34(3), 663-704.

Escanciano, J. C. (2006a), "A consistent diagnostic test for regression models using projections," Econometric Theory, 22, 1030-1051.

Escanciano, J. C. (2006b), "Goodness-of-Fit Tests for Linear and Nonlinear Time Series Models," Journal of the American Statistical Association, 101(474), 531-541.

Escanciano, J. C. (2008), "Joint and marginal specification tests for conditional mean and variance models," Journal of Econometrics, 143(1), 74-87.

Freyberger, J., and Rai, Y. (2018), "Uniform confidence bands: characterization and optimality," Journal of Econometrics, Forthcoming. 
Giné, E., and Zinn, J. (1990), "Bootstrapping general empirical measures," The Annals of Probability, 18(2), 851-869.

González-Manteiga, W., and Crujeiras, R. M. (2013), "An updated review of Goodness-of-Fit tests for regression models," Test, 22(3), 361-411.

Goodman-Bacon, A. (2017), "Difference-in-Differences with Variation in Treatment Timing," Working Paper, .

Heckman, J. J., Ichimura, H., Smith, J., and Todd, P. (1998), "Characterizing selection bias using experimental data," Econometrica, 66(5), 1017-1098.

Heckman, J. J., Ichimura, H., and Todd, P. (1997), "Matching as an econometric evaluation estimator: Evidence from evaluating a job training programme," The Review of Economic Studies, 64(4605-654).

Horvitz, D. G., and Thompson, D. J. (1952), "A Generalization of Sampling Without Replacement From a Finite Universe," Journal of the American Statistical Association, 47(260), 663-685.

Jacobson, L. S., Lalonde, R. J., and Sullivan, D. G. (1993), "Earnings Losses of Displaced Workers," American Economic Review, 83(4), 685-709.

Jardim, E., Long, M., Plotnick, R., van Inwegen, E., Vigdor, J., and Wething, H. (2017), Minimum Wage Increases, Wages, and Low-Wage Employment: Evidence from Seattle,, Technical report, National Bureau of Economic Research, Cambridge, MA.

Kline, P., and Santos, A. (2012), "A Score Based Approach to Wild Bootstrap Inference," Journal of Econometric Methods, 1(1), 1-40.

Kosorok, M. R. (2008), Introduction to empirical processes and semiparametric inference, New York, NY: Springer.

MacKinnon, J. G., and Webb, M. D. (2016), "Difference-in-Differences Inference with Few Treated Clusters," Queen's Economics Department Working Papers No. 1355, pp. 1-44.

MacKinnon, J. G., and Webb, M. D. (2017), "The wild bootstrap for few (treated) clusters," The Econometrics Journal, 20, 1-22.

Mammen, E. (1993), "Bootstrap and wild bootstrap for high dimensional linear models," The Annals of Statistics, 21(1), 255-285.

Meer, J., and West, J. (2016), "Effects of the Minimum Wage on Employment Dynamics," Journal of Human Resources, 51(2), 500-522.

Montiel Olea, J. L., and Plagborg-Møller, M. (2017), "Simultaneous Confidence Bands: Theory, Implementation, and an Application to SVARs," Working Paper, pp. 1-64.

Neumark, D., Salas, J. M., and Wascher, W. (2014), "Revisiting the minimum wage-employment debate: Throwing out the baby with the bathwater?," Industrial and Labor Relations Review, 67(SUPPL), 608-648.

Neumark, D., and Wascher, W. (1992), "Evidence on Employment Effects of Minimum Wages 
and Subminimum Wage Provisions from Panel Data on State Minimum Wage Laws," Industrial and Labor Relations Review, 46(1), 55-81.

Neumark, D., and Wascher, W. (2000), "Minimum Wages and Employment: A Case Study of the Fast-Food Industry in New Jersey and Pennsylvania: Comment," American Economic Review, 90(5), 1362-1396.

Neumark, D., and Wascher, W. L. (2008), Minimum Wages, Cambridge, MA: The MIT Press.

Oreopoulos, P., von Wachter, T., and Heisz, A. (2012), "The Short- and Long-Term Career Effects of Graduating in a Recession," American Economic Journal: Applied Economics, 4(1), 1-29.

Qin, J., and Zhang, B. (2008), "Empirical-Likelihood-Based Difference-in-Differences Estimators," Journal of the Royal Statistical Society. Series B (Methodological), 75(8), 329-349.

Sant'Anna, P. H. C. (2016), "Program Evaluation with Right-Censored Data," ArXiv preprint arXiv:1604.02642, .

Sant'Anna, P. H. C. (2017), "Nonparametric Tests for Treatment Effect Heterogeneity with Duration Outcomes," Arxiv preprint arXiv:1612.02090, .

Sant'Anna, P. H. C., and Song, X. (2018), "Specification Tests for the Propensity Score," Arxiv preprint arXiv:1611.0621\%, .

Schmitt, J. (2013), "Why Does the Minimum Wage Have No Discernible Effect on Employment?," Center for Economic and Policy Research, (February), 1-28.

Sherman, M., and Le Cessie, S. (2007), "A comparison between bootstrap methods and generalized estimating equations for correlated outcomes in generalized linear models," Communications in Statistics - Simulation and Computation, 26(3), 901-925.

Słoczyński, T. (2017), "A General Weighted Average Representation of the Ordinary and TwoStage Least Squares Estimands," Working Paper, .

Stinchcombe, M. B., and White, H. (1998), "Consistent specification testing with nuisance parameters present only under the alternative," Econometric Theory, 14, 295-325.

Stute, W. (1997), "Nonparametric model checks for regression," The Annals of Statistics, 25(2), 613-641.

van der Vaart, A. W. (1998), Asymptotic Statistics, Cambridge: Cambridge University Press.

van der Vaart, A. W., and Wellner, J. A. (1996), Weak Convergence and Empirical Processes, New York: Springer.

Wooldridge, J. M. (2005), "Fixed-Effects and Related Estimators for Correlated RandomCoefficient and Treatment-Effect Panel Data Models," Review of Economics and Statistics, $87(2), 385-390$. 